\title{
A theory for pressures in cylindrical silos under concentric mixed flow
}

\author{
Adam J. Sadowski ${ }^{1}$, J. Michael Rotter ${ }^{2} \&$ Jørgen Nielsen ${ }^{3}$
}

\begin{abstract}
This paper presents a theory for the prediction of pressures in circular silos under concentric mixed flow, assuming an internal flow channel of conical profile with straight but inclined sides. The theory is based on a generalised application of the classical method of 'slice equilibrium' together with additional assumptions based on a treatment of the granular solid as a Coulombic material. Only one of the resulting pair of coupled linear ordinary differential equations may be solved in closed form, while both numerical and approximate closed-form solutions are explored for the other. The derivation of the theory is presented in full and a series of parametric studies explores the predictions and compares these with qualitative observations from experiments. In particular, the significant overpressure that is known to occur at the 'effective transition', where the internal flow channel intersects with the silo wall, may be estimated quantitatively for the first time.
\end{abstract}

\section{Keywords}

Granular solids, silo pressures, concentric mixed flow, straight-sided channels, internal hopper, discharge overpressures.

${ }^{1}$ Senior Lecturer in Structural Engineering, Department of Civil and Environmental Engineering, Imperial College London, UK.

${ }^{2}$ Visiting Professor of Civil Engineering, Department of Civil and Environmental Engineering, Imperial College London (also Emeritus Professor of Civil Engineering, Institute for Infrastructure and Environment, University of Edinburgh, UK).

3 Honorary Professor, Danish Building Research Institute, Aalborg University, Denmark. 


\section{Nomenclature}

\begin{tabular}{|c|c|c|}
\hline & [L] & Radius of the cylindrical silo \\
\hline$h_{c}$ & [L] & Height of the cylindrical silo from its base to the effective surface \\
\hline$m$ & {$[-]$} & Dimensionless group for the stationary granular solid \\
\hline$n$ & {$[-]$} & Dimensionless group for the flowing granular solid \\
\hline$p_{\text {hce }}$ & {$\left[\mathrm{F} . \mathrm{L}^{-2}\right]$} & Mean horizontal pressure in the flowing granular solid \\
\hline$p_{\text {hce, },}$ & {$\left[\mathrm{F} . \mathrm{L}^{-2}\right]$} & The same as $p_{\text {hce }}$ except defined in terms of the $z$ coordinate \\
\hline$p_{\text {hceT }}$ & {$\left[\right.$ F.L. $\left.{ }^{-2}\right]$} & The value of $p_{\text {hce }}$ at the effective transition \\
\hline$p_{\text {hse }}$ & {$\left[\right.$ F.L.- $\left.{ }^{-2}\right]$} & Mean horizontal pressure in the stationary granular solid \\
\hline$p_{\text {hseT }}$ & {$\left[\mathrm{F} . \mathrm{L}^{-2}\right]$} & The value of $p_{h s e}$ at the effective transition \\
\hline$p_{n e}$ & {$\left[\mathrm{~F} . \mathrm{L}^{-2}\right]$} & $\begin{array}{l}\text { Mean normal pressure at the inclined interface between the flowing and } \\
\text { stationary granular solids }\end{array}$ \\
\hline$p_{n e T}$ & {$\left[\mathrm{~F} . \mathrm{L}^{-2}\right]$} & The value of $p_{n e}$ at the effective transition \\
\hline$p_{t e}$ & {$\left[\mathrm{~F} . \mathrm{L}^{-2}\right]$} & $\begin{array}{l}\text { Mean frictional traction at the inclined interface between the flowing and } \\
\text { stationary granular solids }\end{array}$ \\
\hline$p_{t e T}$ & {$\left[\right.$ F.L.- $\left.{ }^{-2}\right]$} & The value of $p_{t e}$ at the effective transition \\
\hline$p_{v c e}$ & {$\left[\mathrm{~F} . \mathrm{L}^{-2}\right]$} & Mean vertical pressure in the flowing granular solid \\
\hline$p_{v c e, x}$ & {$\left[\right.$ F.L.- $\left.{ }^{-2}\right]$} & The same as $p_{v c e}$ except defined in terms of the $x$ coordinate \\
\hline$p_{v c e, z}$ & {$\left[\mathrm{~F} . \mathrm{L}^{-2}\right]$} & The same as $p_{v c e}$ except defined in terms of the $z$ coordinate \\
\hline$p_{v c e T}$ & {$\left[\mathrm{~F} . \mathrm{L}^{-2}\right]$} & The value of $p_{v c e}$ at the effective transition \\
\hline$p_{v s e}$ & {$\left[\mathrm{~F} . \mathrm{L}^{-2}\right]$} & Mean vertical pressure in the stationary granular solid \\
\hline$p_{v s e T}$ & {$\left[\mathrm{~F} . \mathrm{L}^{-2}\right]$} & The value of $p_{v s e}$ at the effective transition \\
\hline$x$ & {$[\mathrm{~L}]$} & Upwards-positive vertical coordinate, zero at the effective outlet \\
\hline$x_{T}$ & [L] & Height of the effective transition above the effective outlet \\
\hline$z$ & [L] & Downwards-positive vertical coordinate, zero at the effective surface \\
\hline$z_{T}$ & [L] & Depth of the effective transition below the effective surface \\
\hline$C_{h}$ & {$[-]$} & $\begin{array}{l}\text { Excess in horizontal pressure at the effective transition under mixed flow } \\
\text { relative to mass flow }\end{array}$ \\
\hline$C_{w}$ & {$[-]$} & Excess in accumulated wall friction under mixed flow relative to mass flow \\
\hline$F_{e}$ & {$[-]$} & $\begin{array}{l}\text { Discharge pressure ratio for the inclined interface between the flowing and } \\
\text { stationary granular solids }\end{array}$ \\
\hline$F_{t}$ & {$[-]$} & $\begin{array}{l}\text { Ratio of the net outward force under mixed flow relative to that under mass } \\
\text { flow, as integrated from the effective transition to the 'crossover' depth }\end{array}$ \\
\hline$G_{t}$ & {$[-]$} & $\begin{array}{l}\text { Ratio of the gradient of the horizontal pressure just below the effective } \\
\text { transition under mixed flow relative to that under mass flow }\end{array}$ \\
\hline$K_{e, c w}$ & {$[-]$} & $\begin{array}{l}\text { Discharge pressure ratio for the vertical interface between the silo wall and } \\
\text { flowing granular solid }\end{array}$ \\
\hline$K_{e, s w}$ & {$[-]$} & $\begin{array}{l}\text { Discharge pressure ratio for the vertical interface between the silo wall and } \\
\text { stationary granular solid }\end{array}$ \\
\hline$S_{t}$ & {$[-]$} & $\begin{array}{l}\text { Normalised 'crossover' depth of the intersection of the horizontal pressure } \\
\text { distribution under mixed flow with the Janssen distribution under mass flow }\end{array}$ \\
\hline$\beta$ & {$[-]$} & Angle of inclination of the internal conical flow channel \\
\hline$\gamma$ & {$\left[\mathrm{F} . \mathrm{L}^{-3}\right]$} & Unit weight for the bulk granular solid \\
\hline$\theta_{c r}$ & {$[-]$} & $\begin{array}{l}\text { Hypothesised critical value of combined interface roughness and incline at } \\
\text { which an internal rupture layer forms inside the flowing granular solid }\end{array}$ \\
\hline$\mu_{i}$ & {$[-]$} & $\begin{array}{l}\text { 'Ideally rough' friction coefficient at the inclined interface between the flowing } \\
\text { and stationary granular solids }\end{array}$ \\
\hline$\mu_{w}$ & {$[-]$} & $\begin{array}{l}\text { 'Fully developed' friction coefficient at the vertical interface between the silo } \\
\text { wall and the granular solid (flowing or stationary) }\end{array}$ \\
\hline$\phi_{i}$ & {$[-]$} & Angle of internal friction of the granular solid \\
\hline$\phi_{w}$ & [-] & Angle of friction at a potentially inclined interface between two solids \\
\hline
\end{tabular}




\section{Introduction}

The evaluation of pressures caused by discharging granular solid from a cylindrical silo continues to pose a significant scientific challenge even in the modern era of advanced measuring equipment and access to high computing power. The current Eurocode on actions on silos EN 1991-4 (2006) considers three nominally concentric discharge flow patterns. The entire stored solid is in motion during 'mass flow' (Fig. 1a). Under 'mixed flow', a channel of flowing material expands and reaches the silo wall significantly below the surface of the stored material at a location known as the 'effective transition' (Fig. 1b). If the channel is so steep that it does not intersect with the silo wall (Fig. 1c), 'pipe flow' occurs which is known to cause only very moderate increases in loads during discharge (Munch-Andersen and Nielsen, 1990; Nielsen, 1998). However, the pressure increase at the effective transition under mixed flow may be quite large depending on how the internal flow channel expands from the outlet. The current EN 1991-4 (2006) attempts to account for this in a design assessment through empirical 'patch' load devices, but these are based on past experience with little rigorous theoretical background. This paper aims to provide this theoretical background.

a) Mass flow

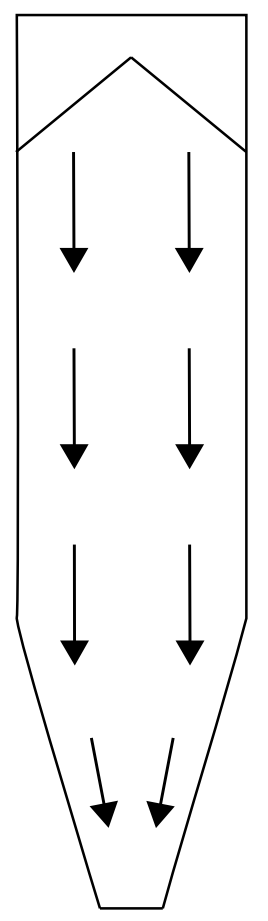

b) Mixed flow

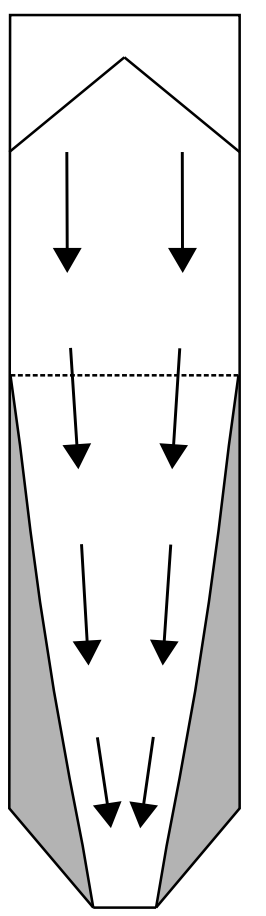

c) Pipe flow

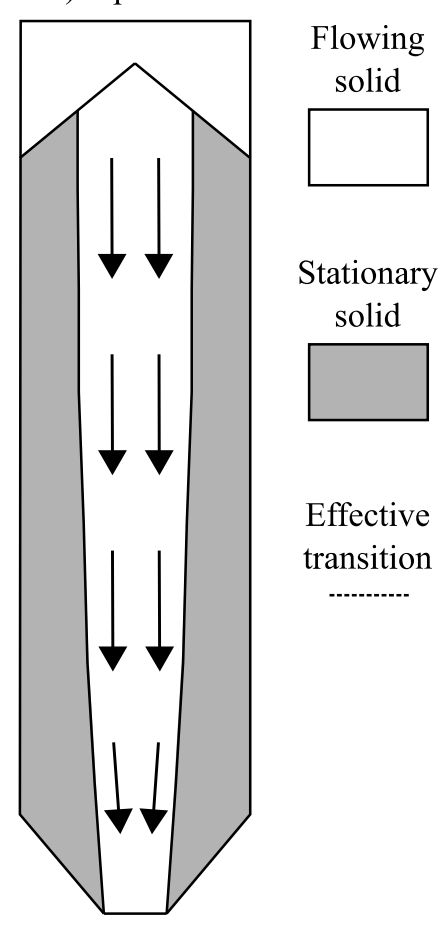

Fig. 1 - Schematic of nominally concentric flow patterns after EN 1991-4 (2006). 
Mass flow design of silos for coarse-grained materials without a fluidized bottom or other flow aids is only possible when the bottom part has inclined walls, for circular silos typically a conical hopper. In such silos, classical theories for flow and pressures already exist (e.g. Jenike, 1961, 1964; Walker, 1966; Walters, 1973; Nedderman, 1992), and they are not explored further here. However, the formation of internal 'pipes' during flow is known to depend on parameters that cannot easily be incorporated in an equilibrium-based algebraic theory (Nielsen, 1998), and the prediction of the shape of a flow channel is therefore not a part of this study. For example, pipe flow is known to be more likely for densely-packed solids which require extensive dilation to flow that only a vertical free-fall through an outlet can provide (Zhong et al., 2001). Conversely, flow of a loosely-packed granular solid does not require high dilation and wider 'mixed' flow channels are possible. A shift may even be seen during the discharge period, where the onset of flow may be in pipe flow, but where mixed flow may develop during the discharge period.

A detailed review of modern and historical experimental and analytical research on flow pattern, stress and velocity profiles may be found in Saleh et al. (2018) and the narrative will not be reproduced here. However, these authors did report that horizontal overpressures in the order of two have been observed for silos which exhibit an effective transition (e.g. Khelil, 1989; Khelil and Roth, 1990). It will be shown in what follows that the discharge overpressure predictions of the mixed flow theory are commensurate with observations from these and other past experiments (e.g. Nielsen and Andersen, 1981; Hartlén et al., 1984), despite the present theory having clear foundations in a quasi-static Janssen-type classical treatment.

Lastly, for rough-walled silos the conventional thinking is arguably that where a stored granular material slides along a rough wall the particles closest to the wall move while a wall friction coefficient close to the internal angle of friction is developed. This thinking is strongly challenged by the theory presented here, which instead indicates that under passive stress states particles closest to the wall do not move and a much lower friction coefficient exists at what is then an 'ideally rough' interface than is currently assumed. This result is supported by observations of a 'rupture layer' in rough-walled silos (Munch-Andersen and Nielsen, 1990), as further discussed in this paper. 


\section{An equilibrium theory for concentric mixed flow 2.1 System geometry and boundary conditions}

A silo of radius $r$ and total cylindrical height $h_{c}$ under concentric mixed flow is idealised as shown in Fig. 2, using notation that is as far as possible commensurate with that used in the European Standard for actions on silos and tanks EN 1991-4 (2006). For reasons that will be clarified shortly, it is convenient to work with two coordinate systems to locate a horizontal plane through the silo: a downwards-positive $z$ coordinate assumed to be zero at an 'effective surface' of the stored granular solid at the top of the silo, and an upwards-positive $x$ coordinate assumed to be zero at an 'effective outlet' at the bottom of the silo. The two are related by $z=h_{c}-x$. Concentric mixed flow discharge is here characterised by an axisymmetric 'effective transition' at a depth $z_{T}$ or height $x_{T}$ above which the granular solid is entirely in motion and below which the granular solid only flows within a conical channel with apex half-angle $\beta$ to the vertical and surrounded by stationary material. These two regions of flow are here termed the 'plug flow' ( $0 \leq z \leq z_{T}$ or $\left.x_{T} \leq x \leq h_{c}\right)$ and 'internal hopper flow' $\left(z_{T} \leq z \leq h_{c}\right.$ or $\left.0 \leq x \leq x_{T}\right)$ regions, and it is convenient to work with these in terms of the $z$ and $x$ coordinate systems respectively.

The mean vertical pressures over the cross-sections within the flowing and stationary solid regions are identified by $p_{v c e}$ and $p_{v s e}$ respectively. It is also convenient to further qualify the pressure component in the flowing material as $p_{v c e, z}$ and $p_{v c e, x}$, depending on whether it refers to the 'plug flow' or the 'internal hopper' regions respectively. Due to the absence of stationary solid within the 'plug flow' region, the associated pressure component $p_{v s e}$ is not defined here and the region is characterised by a single linear differential equation in $p_{v c e, z}$ requiring only one equilibrium boundary condition. It is assumed that the silo is sufficiently slender to permit the classical Janssen boundary condition of zero mean vertical pressure at the top surface to be adopted as a boundary condition in the 'plug flow' region:

BC_MF_1 : $p_{v c e, z}(0)=0$

The 'internal hopper' region contains both pressure components and is characterised by a system of two coupled linear differential equations in $p_{v c e, x}$ and $p_{v s e}$, requiring a total of two equilibrium boundary conditions. It is assumed that $p_{v c e, x}$ and $p_{v c e, z}$ must be equal at the effective transition, such that: 
BC_IH_1: $p_{v c e, x}\left(x_{T}\right)=p_{v c e, z}\left(z_{T}\right) \equiv p_{v c e T}$

It is further asserted that the starting value of the $p_{v s e}$ at the effective transition, or $p_{v s e T}$, may be deduced from $p_{v c e T}$ by considering local equilibrium (presented shortly):

BC_IH_2 : $p_{v s e T}=f\left(p_{v c e T}\right)$

For simplicity, the effective outlet is considered to be coincident with the apex of the 'internal hopper', consistent with assumptions made elsewhere (Dąbrowski, 1957; Walker, 1966; Walters, 1973; Hampe, 1987; Nedderman, 1992). For slender silos, the loss of accuracy associated with neglecting a finite-size outlet orifice is minimal.

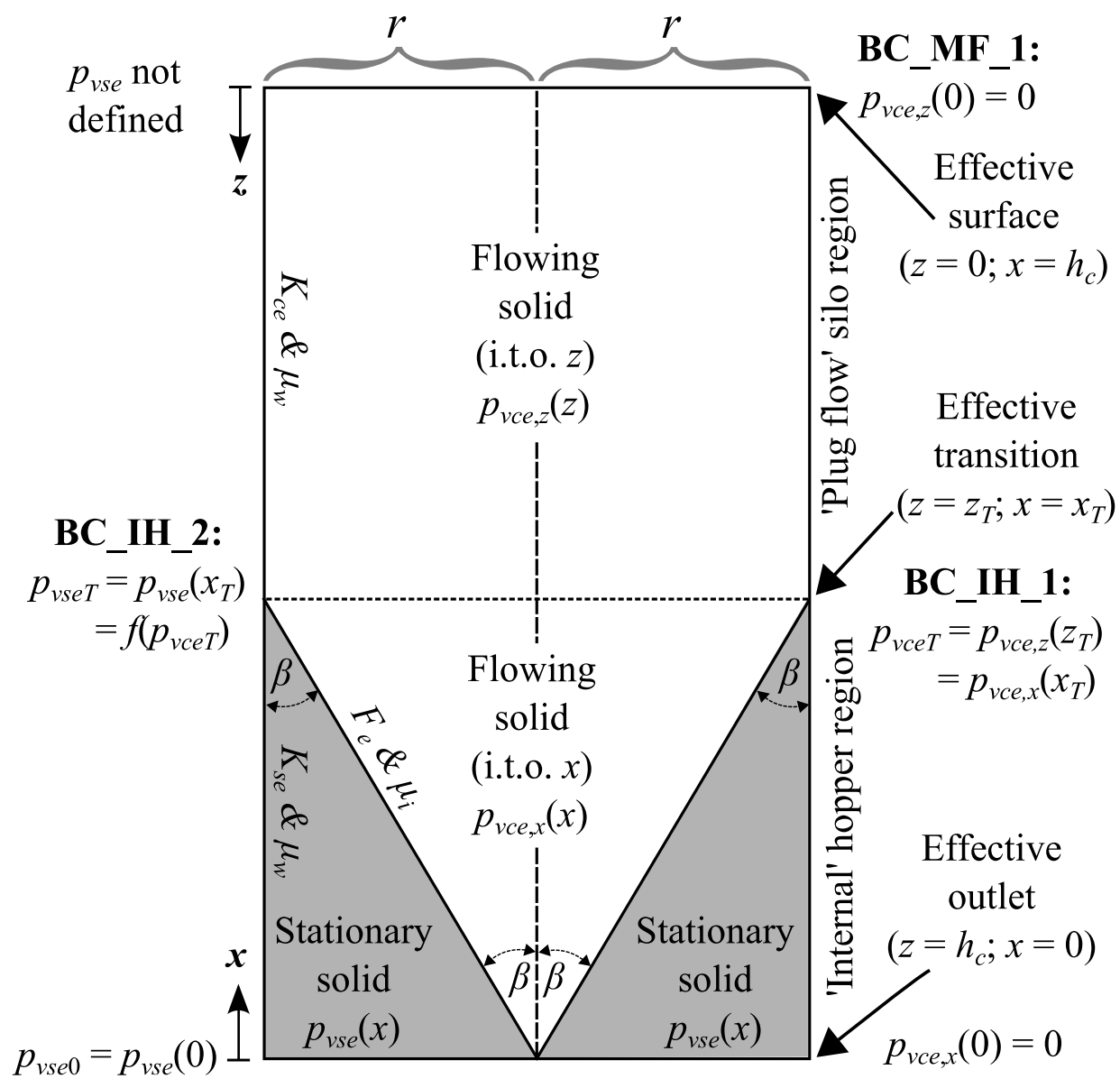

Fig. 2 - Flow regions and coordinate system definition. 


\subsection{Slice equilibrium}

\subsubsection{Material relations}

In slice equilibrium models, it is classically assumed that the mean horizontal pressure $p_{h}$ acting against a vertical wall at any level is related to the mean vertical pressure $p_{v}$ within the granular solid by a lateral pressure ratio $K$. In the 'plug flow' region (Fig. 2) the relationship is here taken as $p_{h c e, z}=K_{e, c w} \cdot p_{v c e, z}$ while in the stationary solid in the 'internal hopper' region it is taken as $p_{h s e}=K_{e, s w} \cdot p_{v s e}$, with constants $K_{e, c w}$ and $K_{e, s w}$ representing discharge lateral pressure ratios for the two parts of the wall. Similarly, the mean normal pressure $p_{n e}$ acting against the inclined interface of the flowing and stationary solids within the 'internal hopper' region is related to the mean vertical pressure $p_{v c e, x}$ within the flowing solid by a discharge wall pressure ratio $F_{e}$. The frictional traction at this inclined interface is assumed to be obtained by $p_{t e}=\mu_{i} \cdot p_{n e}$, with a friction coefficient $\mu_{i}$ chosen to appropriately represent the 'rough' nature of this interface. The wall friction between the granular solid and the silo wall is assumed to be fully developed, giving uniform frictional tractions $p_{t c e, z}=\mu_{w} \cdot p_{h c e, z}$ and $p_{t s e}=\mu_{w} \cdot p_{h s e}$, where $\mu_{w}$ is a constant fully developed wall friction coefficient. The equilibrium derivations which follow are independent of the choices for $K_{e, s w}, K_{e, c w}, F_{e}$ and $\mu_{i}$ or whether these are functions of the axial coordinate, but careful derivations for these will be presented shortly. Only vertical equilibrium is considered in what follows.

\subsubsection{Equilibrium within the 'plug flow' region}

An infinitesimally thin slice of thickness $d z$ through the granular solid in the "plug flow' region is presented in Fig. 3. It is assumed that the mean vertical pressure in the flowing solid $p_{v c e, z}$ picks up an infinitesimal increment $d p_{v c e, z}$ across the slice due to the presence of the frictional tractions $p_{w c e, z}$ and the self-weight (where $\gamma$ is the bulk solid density, assumed in the solution to be constant throughout the silo). Implementing the material assumptions leads to the following equation for vertical equilibrium in the slice:

$$
\frac{d p_{v c e, z}}{d z}+\frac{p_{v c e, z}}{z_{o}}=\gamma \text { where } z_{o}=\frac{r}{2 \mu_{w} K_{e, c w}}
$$

This is in fact the Janssen differential equation with $z_{o}$ as the characteristic depth, which has the following solution for the mean vertical pressure assuming a stress-free effective 
surface (BC_MF_1; Eq. 1) and constant material properties:

$p_{v c e, z}(z)=\gamma z_{o}\left(1-e^{-z / z_{o}}\right)$

The mean vertical pressure within the flowing solid at the top of the 'internal hopper (BC_IH_1; Eq. 2) is thus:

$p_{v c e T}=\gamma z_{o}\left(1-e^{-z_{T} / z_{o}}\right)$

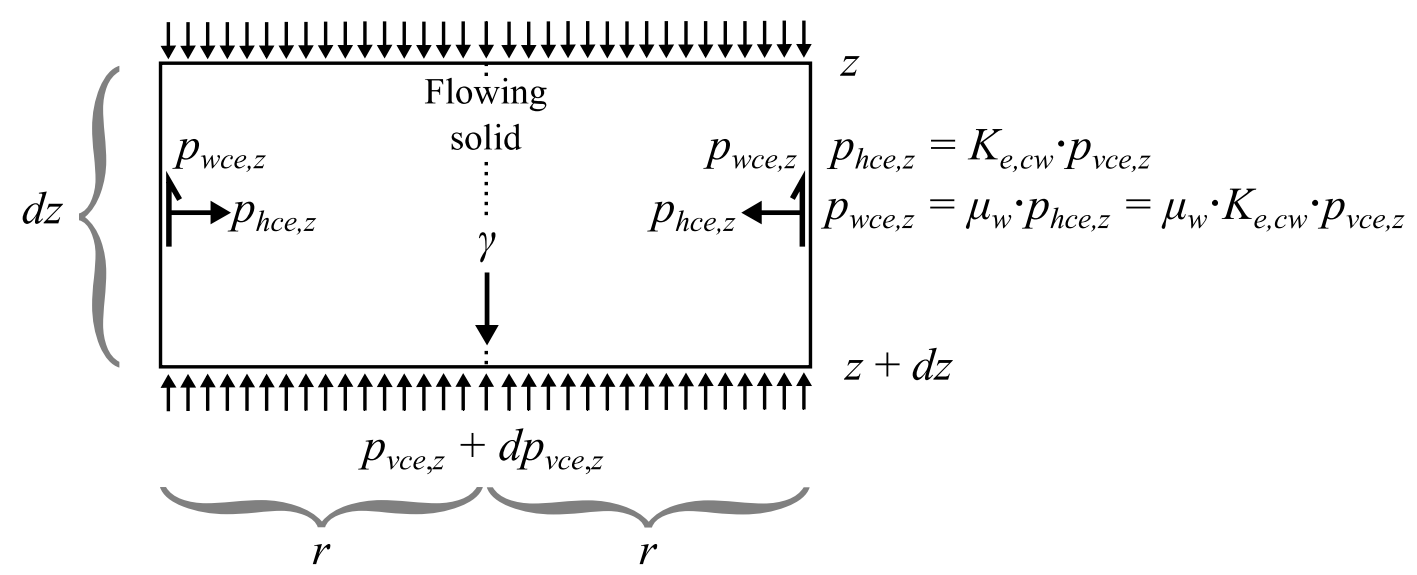

Fig. 3 - Equilibrium on a cylindrical silo element in the 'mass flow' region (horizontal shear stresses at $z$ and $z+d z$ are neglected).

\subsubsection{Equilibrium within the stationary solid at the effective transition}

There is experimental evidence (Hartlén et al., 1984; Munch-Andersen and Nielsen, $1989 ; 1990)$ to suggest that the silo wall experiences an abrupt rise in horizontal pressure at the effective transition where the flow channel intersects with the silo. The full equilibrium analysis of the 'internal hopper' region is presented shortly, but a specialised consideration of the equilibrium condition at the effective transition permits a direct exploration of any possible horizontal overpressure at this location while also furnishing the boundary condition for $p_{v s e}(x)$. An infinitesimally thin slice of thickness $d x$ just below the effective transition at $x=x_{T}$ is shown in Fig. 4.

Considering vertical equilibrium within the small wedge of stationary solid only:

$\sum \downarrow: \quad\left(p_{\text {teT }} \cos \beta+p_{\text {neT }} \sin \beta\right) S_{c}+\gamma V_{s}$

$\sum \uparrow: \quad p_{v s e T} A_{s, x-x_{T}}+p_{\text {wseT }} S_{s}$ 
where, ignoring all terms $\mathrm{O}\left(d x^{2}\right)$ or above: $\begin{aligned} & A_{s, x-x_{T}}=2 \pi x_{T} d x \tan ^{2} \beta \quad V_{s}=0 \\ & S_{c}=2 \pi x_{T} d x \frac{\tan \beta}{\cos \beta} \quad S_{s}=2 \pi x_{T} d x \tan \beta\end{aligned}$

In the above, $A_{s, x-x T}$ is the cross-sectional area of the lower part of the wedge of stationary solid material, $S_{c}$ and $S_{s}$ are the stationary solid's lateral surface areas to the flowing channel and wall respectively, and $V_{S}$ is the (negligibly small) wedge volume. Substituting for the material assumptions, it may be shown that the starting value for $p_{v s e}(x), p_{v s e T}$, is (BC_IH_2; Eqs $\left.3 \& 6\right)$ :

$$
p_{v s e T}=p_{v c e T} \frac{F_{e}\left(1+\mu_{i} \cot \beta\right)}{\left(1+\mu_{w} K_{e, s w} \cot \beta\right)}
$$

The ratio $p_{v s e T} / p_{v c e T}$ may also be rearranged to give the maximum dimensionless horizontal overpressure $C_{h}$ at the effective transition:

$$
C_{h}=\frac{p_{h s e T}}{p_{h c e T}}=\frac{K_{e, s w}}{K_{e, c w}} \cdot \frac{F_{e}\left(1+\mu_{i} \cot \beta\right)}{\left(1+\mu_{w} K_{e, s w} \cot \beta\right)}
$$

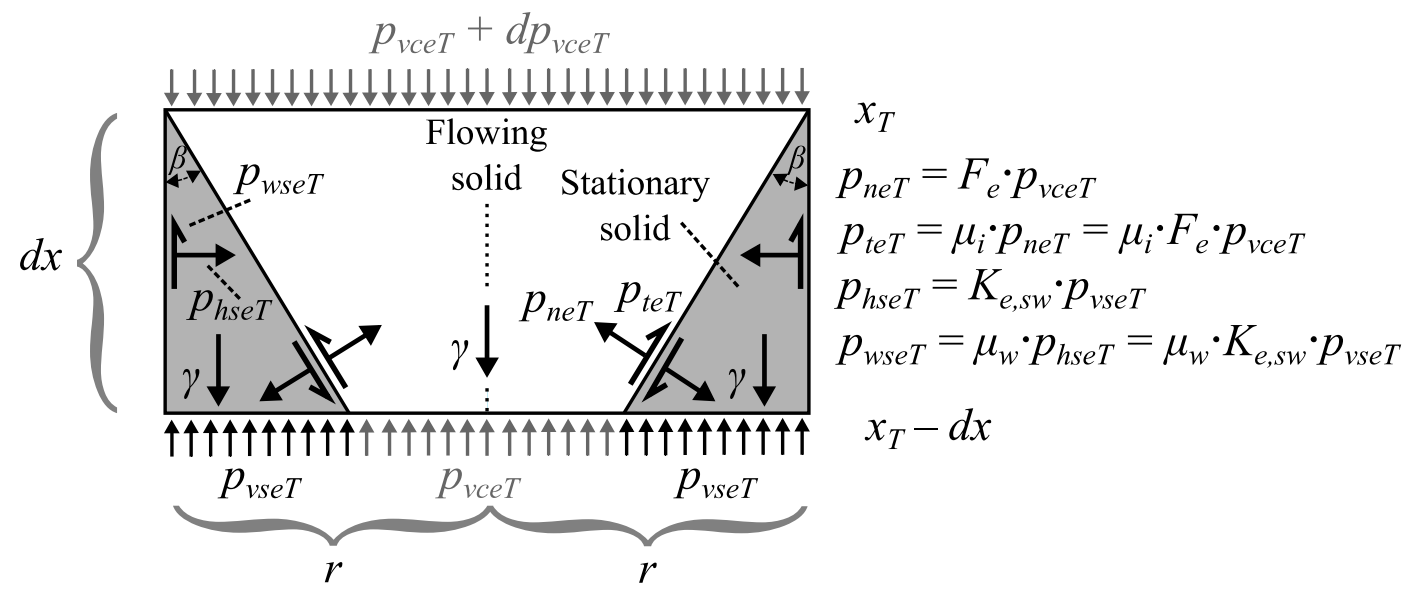

Fig. 4 - Equilibrium on a cylindrical silo element at the effective transition (horizontal shear stresses at $x_{T}$ and $x_{T}-d x$ are neglected).

\subsubsection{Equilibrium within the flowing channel of the 'internal hopper' region}

Directly analogous to the 'plug flow' region, an infinitesimally thin slice of thickness $d x$ through the granular solid in the 'internal hopper' region is presented in Fig. 5. The 
mean vertical pressures in the stationary and flowing solids $p_{v s e}$ and $p_{v c e, x}$ are similarly assumed to pick up an infinitesimal increment $d p_{v s e}$ and $d p_{v c e, x}$ respectively across the slice. Vertical equilibrium within the flowing solid component only, effectively an 'internal hopper', is resolved as follows:

$\sum \downarrow: \quad\left(p_{v c e, x}+d p_{v c e, x}\right) A_{c, x+d x}+\gamma V_{c}$

$\sum \uparrow: \quad p_{v c e, x} A_{c, x}+\left(p_{t e} \cos \beta+p_{n e} \sin \beta\right) S_{c}$

where:

$$
r_{x}=x \tan \beta \quad r_{x+d x}=(x+d x) \tan \beta \quad A_{c, x}=\pi r_{x}^{2} \quad A_{c, x+d x}=\pi r_{x+d x}^{2}
$$

$$
V_{c}=\frac{\pi}{3}\left(r_{x}^{2}+r_{x+d x}^{2}+r_{x} r_{x+d x}\right) d x \quad S_{c}=\pi\left(r_{x}+r_{x+d x}\right) \sqrt{\left(r_{x+d x}-r_{x}\right)^{2}+d x^{2}}
$$

In the above, $r_{x}$ and $r_{x+d x}$ are the flow channel radii at the lower and upper boundaries of the slice with $A_{c}$ and $A_{c, x+d x}$ as the respective cross-sectional areas, while $V_{c}$ and $S_{c}$ are the volume and lateral surface area (interface area between the flowing and stationary solids) of the truncated conical flow channel geometry.

$$
p_{v s e}+d p_{v s e} \quad p_{v c e, x}+d p_{v c e, x} \quad p_{v s e}+d p_{v s e}
$$

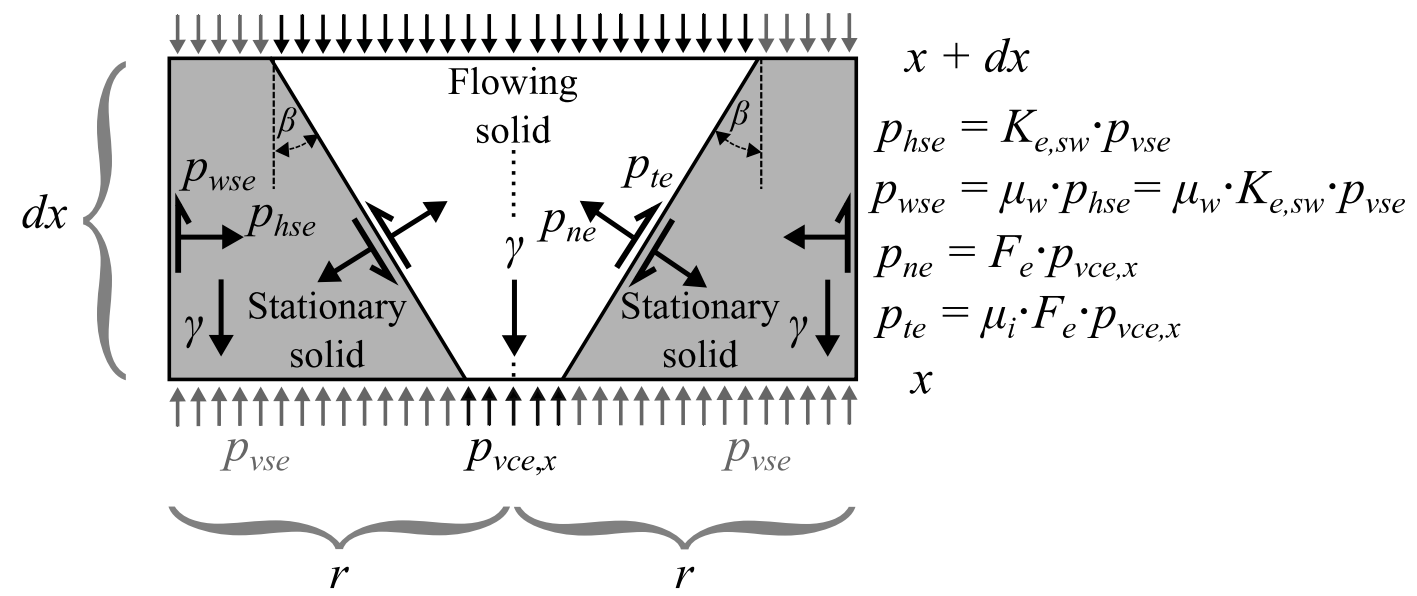

Fig. 5 - Equilibrium on a cylindrical silo element in the 'internal hopper' region (horizontal shear stresses at $x$ and $x+d x$ are neglected).

Implementing the material assumptions and retaining only terms up to $\mathrm{O}(d x)$ leads to a linear differential equation recognisable as that from Walker theory but specialised for an 'ideally rough' hopper (Drescher, 1991; Nedderman, 1992): 
$\frac{d p_{v c e, x}}{d x}-\frac{n}{x} p_{v c e, x}=-\gamma$ where $n=2\left(F_{e}\left(1+\mu_{i} \cot \beta\right)-1\right)$

The solution for the mean vertical pressure, assuming equilibrium with the 'plug flow' region at the effective transition $\left(\mathrm{BC}_{-} \mathrm{IH}_{-} 1\right.$; Eqs 2 \& 6) and constant material properties, is as follows:

$p_{v c e, x}(x)=p_{v c e T}\left(\frac{x}{x_{T}}\right)^{n}+\frac{\gamma x_{T}}{n-1}\left(\frac{x}{x_{T}}-\left(\frac{x}{x_{T}}\right)^{n}\right)$

\subsubsection{Equilibrium within the stationary solid of the 'internal hopper' region}

A similar analysis for the stationary solid component only leads to the following resolution of vertical equilibrium:

$\sum \downarrow: \quad\left(p_{v s e}+d p_{v s e}\right) A_{s, x+d x}+\gamma V_{s}+\left(p_{t e} \cos \beta+p_{n e} \sin \beta\right) S_{c}$

$\sum \uparrow: \quad p_{\text {vse }} A_{s, x}+p_{\text {wse }} S_{s}$

where: $A_{s, x}=\pi r^{2}-A_{c, x} \quad A_{s, x+d x}=\pi r^{2}-A_{c, x+d x}$

$$
V_{s}=V-V_{c} \quad S_{s}=2 \pi r d x
$$

In the above, $A_{s, x}$ and $A_{s, x+d x}$ are the cross-sectional areas of the stationary solid at the lower and upper boundaries of the slice respectively, $V_{s}$ is the slice volume of the stationary solid, $S_{s}$ is its contact area with the silo wall, and remaining symbols have the same meaning as above. Implementing the material assumptions leads to the following linear differential equation ( $n$ is given by Eq. 8 ):

$$
\frac{d p_{v s e}}{d x}-2\left(\frac{x+x_{T} m}{x_{T}^{2}-x^{2}}\right) p_{v s e}=-x\left(\frac{2+n}{x_{T}^{2}-x^{2}}\right) p_{v c e, x}-\gamma \text { where } m=\mu_{w} K_{e, s w} \cot \beta
$$

The coefficients point to a singularity as $x \rightarrow x_{T}$ where the differential equation is no longer defined, but the equilibrium relation in Eq. 7 ensures that the solution $p_{\text {vse }}(x)$ must tend to a finite limit of $p_{v s e T}$ at the effective transition. This differential equation has no closed-form solution for $p_{v s e}(x)$ due a complicated particular integral arising from $p_{v c e, x}$ (Eq. 9), and it is perhaps best solved numerically by a simple Euler integration scheme $p_{v s e^{i+1}}^{i+}=p_{v s e}^{i}-d p_{v s e^{i}}$ using the starting value of $p_{v s e}^{i=0}=p_{v s e T}$ at $x=x_{T}$ and progressing in 
reverse towards $x=0$ (Eq. 7). Alternatively, it may be manipulated to yield the following solution for $p_{v s e}(x)$, assuming constant material properties:

$p_{v s e}(x)=\frac{\left(x_{T}+x\right)^{m-1}}{\left(x_{T}-x\right)^{m+1}} \cdot \frac{1}{(n-1)} \cdot \int_{x}^{x_{T}} I(y) \cdot \frac{\left(x_{T}-y\right)^{m}}{\left(x_{T}+y\right)^{m}} d y$ such that $\lim _{x \rightarrow x_{T}} p_{v s e(x)}=p_{v s e T}$

where $I(y)=\left\{\begin{array}{c}p_{v c e T}\left(\frac{y}{x_{T}}\right)^{n} y(n+2)(n-1) \ldots \\ -\gamma x_{T}\left[\left(\frac{y}{x_{T}}\right)^{n} y(n+2)-3 \frac{y^{2}}{x_{T}}-x_{T}(n-1)\right]\end{array}\right\}$

The integral in Eq. 11 is here expressed in terms of a 'dummy' variable $y$ and must be evaluated numerically. The derivative of $p_{v s e}(x)$ with respect to $x$ may be written as:

$$
\frac{d p_{v s e}}{d x}=\frac{1}{(n-1)\left(x_{T}^{2}-x^{2}\right)} \cdot\left\{\frac{\left(x_{T}+x\right)^{m}}{\left(x_{T}-x\right)^{m}}\left[\frac{(m+1)}{\left(x_{T}-x\right)}+\frac{(m-1)}{\left(x_{T}+x\right)}\right] \cdot \int_{x}^{x_{T}} I(y) \cdot \frac{\left(x_{T}-y\right)^{m}}{\left(x_{T}+y\right)^{m}} d y-I(x)\right\}
$$

By expanding the integrand as a two-term Taylor series about $x=x_{T}$, it may be shown that the limiting gradient of $p_{v s e}(x)$ at the effective transition is:

$$
\left.\frac{d p_{v s e}}{d x}\right|_{x T}=\lim _{x \rightarrow x_{T}} \frac{d p_{v s e}}{d x}=\frac{p_{v c e T}(n+2)(n m+m+n)-\gamma x_{T}(n+4)(m+1)}{2 x_{T}\left(m^{2}+3 m+2\right)}=-\left.\frac{d p_{v s e}}{d z}\right|_{z T}
$$

A further quantity of interest, here called $G_{T}$, is the ratio of the gradient of the horizontal pressure in the stationary solid at the effective transition $p_{h s e T}=K_{e, s w} \cdot p_{v s e T}$ relative to the gradient that would exist in a flowing solid at that location under Janssen conditions $p_{h c e T}=K_{e, c w} \cdot p_{v c e T}:$

$$
G_{T}=\frac{\left(\left.\frac{d p_{h s e}}{d z}\right|_{z_{T}}\right)}{\left(\left.\frac{d p_{h c e}}{d z}\right|_{z_{T}}\right)}=\frac{K_{e, s w}}{K_{e, c w}} \cdot \frac{\left[\left(h_{c}-z_{T}\right)(n+4)(m+1)-z_{0}\left(1-e^{-z_{T} / z_{0}}\right)(n+2)(n m+m+n)\right]}{2\left(h_{c}-z_{T}\right)\left(m^{2}+3 m+2\right) e^{-z_{T} / z_{0}}}
$$

Values of $G_{T} \gg>0$ represent a horizontal pressure increasing beyond $p_{h s e T}$ with depth and thus a pressure 'bulge' against the wall just below the effective transition (Fig. 6a), 
values of $G_{T} \approx 0$ represent a flat 'plateau' in the horizontal pressure distribution (Fig. 6b) while values of $G_{T}<<0$ (including very negative ones) instead represent a sharp 'drop' in horizontal pressure from $p_{\text {hseT }}$ (Fig. 6c). The discriminating condition is:

$$
\frac{\left(h_{c}-z_{T}\right)}{z_{0}\left(1-e^{-z_{T} / z_{0}}\right)} \cdot \frac{(n+4)(m+1)}{(n+2)(n m+m+n)} \rightarrow\left\{\begin{array}{cc}
>1 & \text { bulge }\left(G_{T}>0\right) \\
\approx 1 & \text { plateau }\left(G_{T} \approx 0\right) \\
<1 & \text { drop }\left(G_{T}<0\right)
\end{array}\right.
$$

'Pointed' horizontal pressure distributions with steep drops become more likely for rougher walls (high $\mu_{w}$ ) or stronger solids (high $\phi_{i}$ ) as well as for effective transitions that occur lower in the silo $\left(z_{T} \rightarrow h_{c}\right)$.

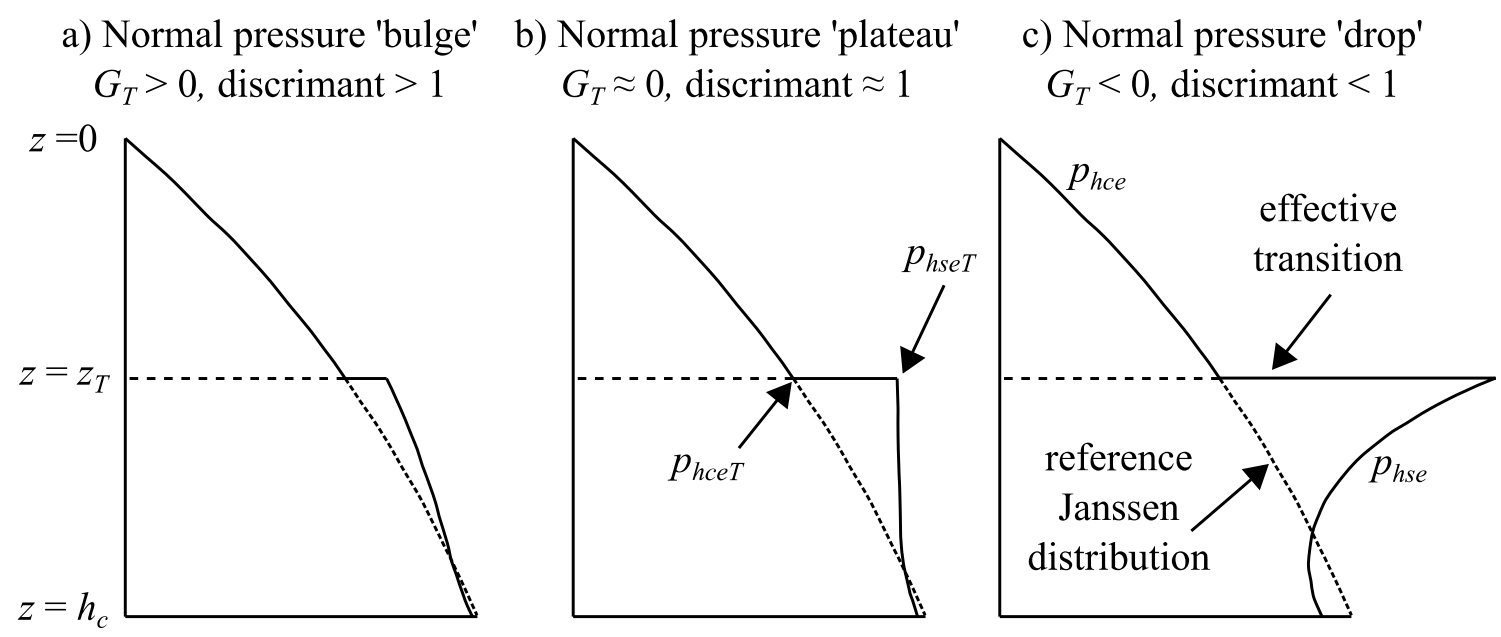

Fig. 6 - Schematics of typical horizontal pressure distributions depending on the conditions at the effective transition.

\subsection{Mohr-Coulomb failure criteria}

\subsubsection{Nä̈ve analysis}

The granular solid is treated here as a cohesionless Coulombic material characterised by an angle of internal friction $\phi_{i}$. The classical interpretation of the stress state in a granular solid adjacent to a wall of with a fully developed wall friction coefficient $\mu_{w}=$ $\tan \phi_{w}$, assuming the solid is simultaneously in a state of rupture against the wall (characterised by the Wall Yield Locus, or WYL) and internal rupture (characterised by the Internal Yield Locus, or IYL), is performed with the aid of a Mohr's circle as shown in Fig. 7a. The sign convention of positive compressive stresses and positive anti- 
clockwise shears from soil mechanics is adopted here. The Mohr's circle leads to the following expression for the 'wall pressure ratio' at discharge $p_{n e} / p_{v e}$ :

$$
\frac{p_{n e}}{p_{v e}}=\frac{1+\sin \phi_{i} \cos \left(\omega+\phi_{w}\right)}{1-\sin \phi_{i} \cos \left(\omega+\phi_{w}+2 \beta\right)} \text { where } \omega=\sin ^{-1}\left(\frac{\sin \phi_{w}}{\sin \phi_{i}}\right)
$$

This expression is widely used for steep hoppers under discharge whose derivation may be found in classical textbooks (Hampe, 1987; Nedderman, 1992; EN 1991-4, 2006). It is a natural potential first choice for adoption into the mixed flow theory to describe the conditions with the ideally rough 'internal hopper' (Fig. 4) in the limit as $\phi_{w} \rightarrow \phi_{i}$ :

$$
F_{e}=\lim _{\phi_{w} \rightarrow \phi_{i}} \frac{p_{n e}}{p_{v e}}=\frac{1-\sin ^{2} \phi_{i}}{1+\sin \phi_{i} \sin \left(\phi_{i}+2 \beta\right)}
$$

a) Flowing solid in 'passive' state within a hopper

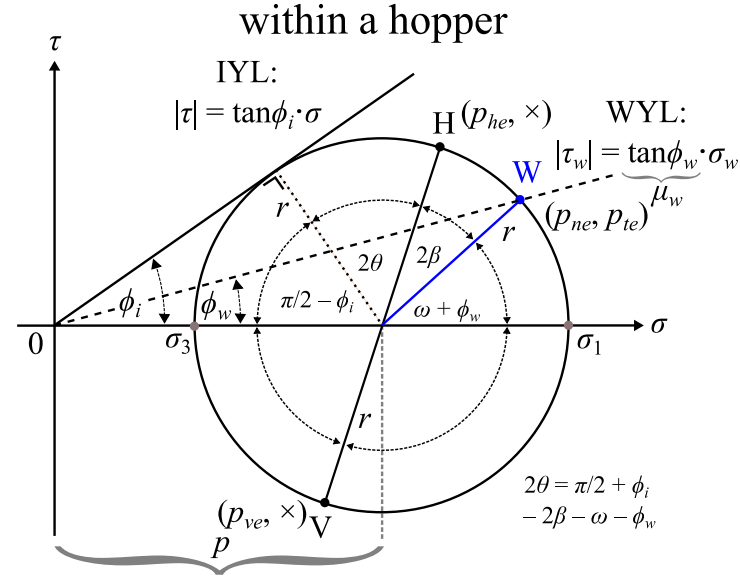

b) Tendency to the incorrect 'active' limit as $\phi_{w} \rightarrow \phi_{i}$

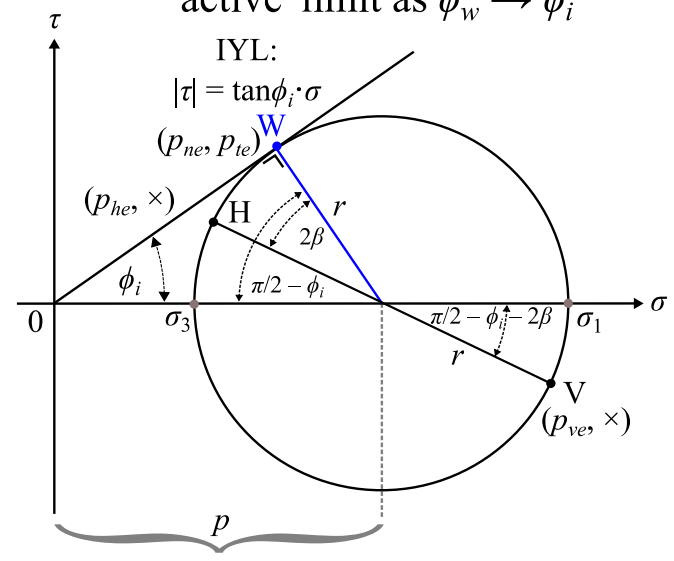

Fig. 7 - Mohr's circle for the stress at the wall in a steep conical hopper at discharge.

However, as the granular solid should arguably be in a 'passive' state (i.e. $p_{h e}>p_{v e}$ ) within the internal flow channel under discharge, the stress state shown in Fig. 7a only satisfies this if

$\omega+\phi_{w}+2 \beta<\frac{\pi}{2}$

as otherwise $p_{h s e}<p_{v s e}$ and the stress state corresponds to an 'active' one. Hoppers which are increasingly shallow $(\beta \uparrow)$ or rough $\left(\omega \uparrow\right.$ and $\left.\phi_{w} \uparrow\right)$ are more likely to violate this 
condition. Unfortunately, according to this reasoning the limiting case of the above system for an 'ideally rough' hopper $\left(\phi_{w} \rightarrow \phi_{i}\right.$; Fig. $\left.7 \mathrm{~b}\right)$ is always 'active', corresponding physically to a rupture plane $\mathrm{W}$ parallel to the 'wall' of the hopper (in the present context this is the inclined interface between the flowing and stationary solids; Fig. 5) as well as a second rupture plane A inclined at $\pi / 2-\phi_{i}$ to W. For this reason, Eq. 17 cannot be used as a starting point for the mixed flow theory.

\subsubsection{Rigorous analysis of the 'active' state}

For completeness, the derivation of the Mohr-Coulomb failure criterion for an 'active' stress state at a wall of arbitrary incline and roughness is briefly reproduced here. According to the system diagram (Fig. 8a), to ensure positive shears on the wall $\mathrm{W}$ and horizontal $\mathrm{H}$ planes as well as WYL < IYL the input parameters must satisfy:

$2 \beta \leq \omega-\phi_{w} \leq \frac{\pi}{2}$ or $0 \leq \omega-\phi_{w}-2 \beta \leq \frac{\pi}{2}-2 \beta$ where $\omega=\sin ^{-1}\left(\frac{\sin \phi_{w}}{\sin \phi_{i}}\right)$

The lateral $K$ and wall pressure $F$ ratios for the 'active' state may be derived as:

$$
K=\frac{1-\sin \phi_{i} \cos \left(\omega-\phi_{w}-2 \beta\right)}{1+\sin \phi_{i} \cos \left(\omega-\phi_{w}-2 \beta\right)}<1 \text { and } F=\frac{1-\sin \phi_{i} \cos \left(\omega-\phi_{w}\right)}{1+\sin \phi_{i} \cos \left(\omega-\phi_{w}-2 \beta\right)}<1
$$

In the limiting case of a vertical wall, under ideally smooth $\left(\phi_{w} \rightarrow 0\right)$ and rough $\left(\phi_{w} \rightarrow\right.$ $\left.\phi_{i}\right)$ the $K$ equation tends to the two well-known results:

$$
\lim _{\substack{\beta \rightarrow 0 \\ \phi_{w} \rightarrow 0}} K=\frac{1-\sin \phi_{i}}{1+\sin \phi_{i}} \text { and } \lim _{\substack{\beta \rightarrow 0 \\ \phi_{w} \rightarrow \phi_{i}}} K=\frac{1-\sin ^{2} \phi_{i}}{1+\sin ^{2} \phi_{i}}
$$

The often-used $K=a K_{0}$ relation, where $a \geq 1$ and $K_{0}=1-\sin \phi_{i}$ is the frictionless 'at rest' ratio from soil mechanics, gives values in-between the above limits (Rotter, 2001). 
a) Solid in 'active' stress state at arbitrary wall roughness and incline

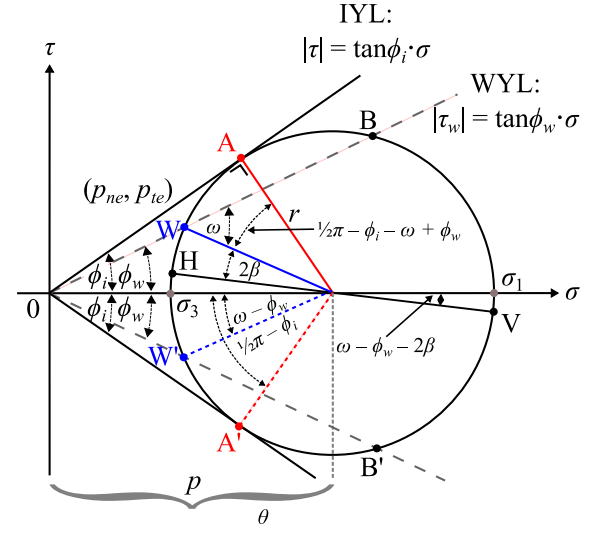

b) Solid in 'passive' state at

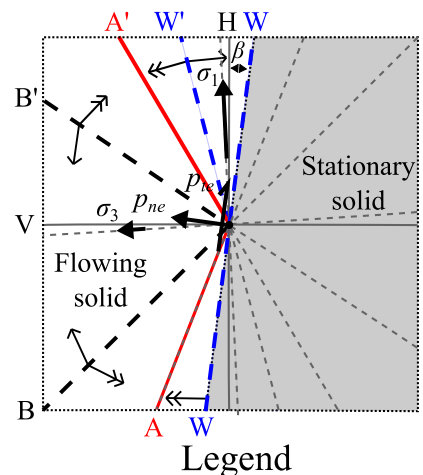
Legend

- rupture plane due to $\phi_{w}$ arbitrary wall roughness and incline - rupture plane due to $\phi_{i}$

('smooth' domain) IYL:

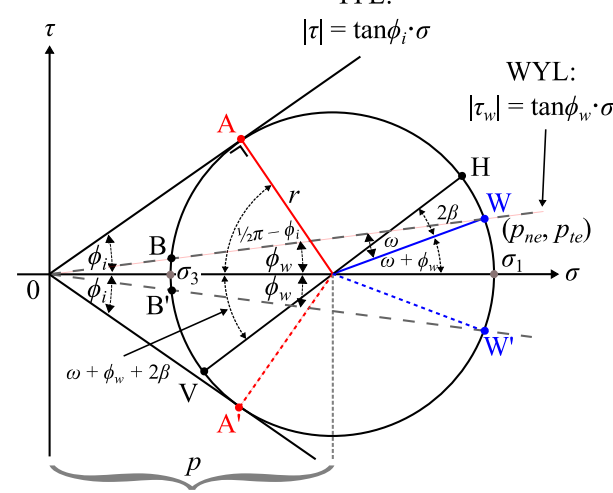

$\longrightarrow$ movement of $\phi_{w}$ rupture plane as $\phi_{w} \rightarrow \phi_{i}$

$\longrightarrow$ movement of $\phi_{w}$ rupture plane as $\phi_{w} \rightarrow 0$

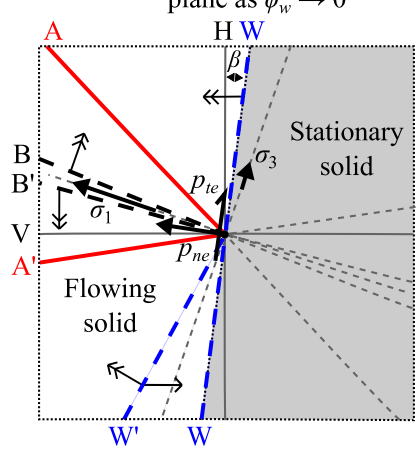

c) Solid in 'passive' state at arbitrary wall roughness and incline

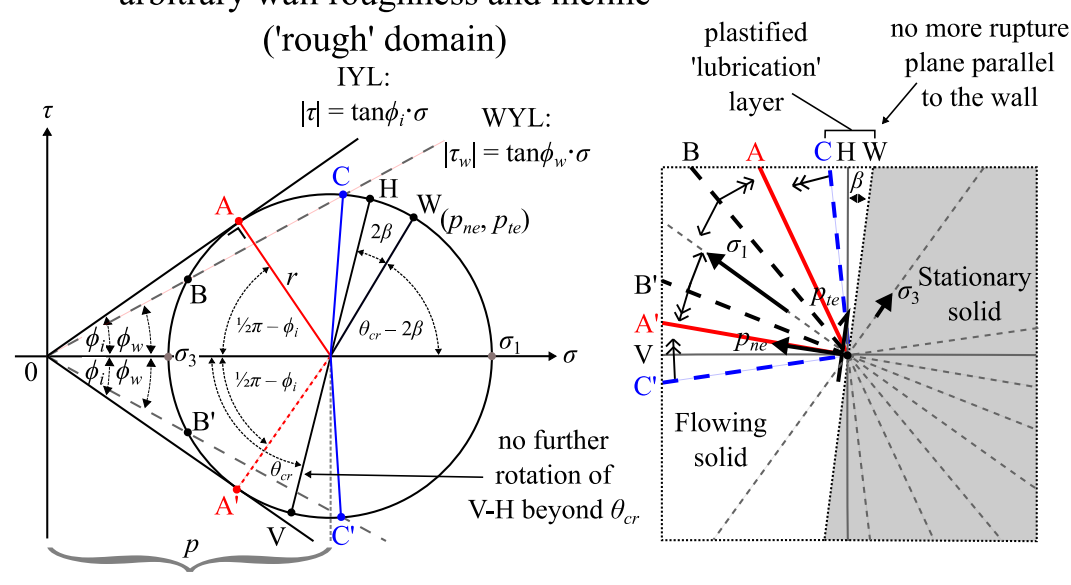

Fig. 8 - Mohr's circles for the stress states and illustrations of failure planes adjacent at an interface of arbitrary incline $\beta$ and roughness $\phi_{w}$ assuming a) 'active', b) 'smooth passive' and c) 'rough passive' states. 


\subsubsection{Rigorous analysis of the 'passive' state}

The discussion in Section 2.3.1 suggests that the derivation of $K$ and $F$ expressions for the 'passive' state that are fully continuous in $\phi_{w}$ and $\beta$ requires special consideration. The Mohr's circle construct is here divided into a 'near-smooth' state (Fig. 8b) where, as long as $0 \leq \phi_{w}<<\phi_{i}$, the granular solid continues to exhibit a rupture layer parallel to the wall with the point W being on the WYL. It is then hypothesised that under increasing wall roughness $\left(\omega \uparrow\right.$ and $\left.\phi_{w} \uparrow\right)$ or incline $(\beta \uparrow)$, at a critical value of $\omega+\phi_{w}+2 \beta=\theta_{c r}$ the rupture plane no longer forms parallel to the wall but within the material itself (point $\mathrm{C} \neq$ $\mathrm{W}$ is on the WYL, but $\mathrm{W}$ is not; Fig. 8c). There is experimental evidence to support this, through observations of the formation of a plastified 'lubrication' layer several particles wide during discharge in rough-walled silos (Munch-Andersen and Nielsen, 1989; 1990). Beyond $\theta_{c r}$, the stress state undergoes no further changes even though $\phi_{w}<\phi_{i}$, suggesting that the ideally rough limiting condition under a 'passive' stress state is achieved at lower values of wall friction than $\phi_{i}$, which may be shown to be given by:

$\mu_{w, c r}=\tan \phi_{w, c r}=\frac{\sin \phi_{i} \sin \left(\theta_{c r}-2 \beta\right)}{1+\sin \phi_{i} \cos \left(\theta_{c r}-2 \beta\right)}$

According to the system diagram (Fig. $8 \mathrm{~b} \& \mathrm{c}$ ), to ensure positive shears on the wall $\mathrm{W}$ and horizontal H planes, WYL < IYL as well as a 'passive' stress state at arbitrary $\phi_{w}$ and $\beta$, the input parameters must always satisfy:

$0 \leq \omega+\phi_{w}+2 \beta \leq \theta_{c r} \leq \frac{\pi}{2}$ where $\omega=\sin ^{-1}\left(\frac{\sin \phi_{w}}{\sin \phi_{i}}\right)$

The 'critical' value rotation of the $\mathrm{V}-\mathrm{H}$ axis to the $\sigma$-axis at which the plastified 'lubrication' layer is assumed to begin forming is not yet known and would require experimental verification. Two potential values, together with their implied conditions on the permissible inclination of the flow channel, proposed at this stage are:

$\theta_{c r}=\left\{\begin{array}{cc}\frac{\pi}{2}-\phi_{i} & \text { H lies opposite A: } \beta<\frac{\pi}{4}-\frac{\phi_{i}}{2} \text { to avoid negative wall shears } \\ \frac{\pi}{2}-\phi_{i}+2 \beta & \text { W lies opposite A: } \beta<\frac{\phi_{i}}{2} \text { to avoid 'active' state }\end{array}\right.$

The lateral $K$ and wall pressure $F$ ratios for the 'passive' state may then be derived as: 
$K=\frac{1+\sin \phi_{i} \cos \theta}{1-\sin \phi_{i} \cos \theta}>1$ and $F=\frac{1+\sin \phi_{i} \cos (\theta-2 \beta)}{1-\sin \phi_{i} \cos \theta}>1$ where $\theta=\min \left\{\begin{array}{c}\omega+\phi_{w}+2 \beta \\ \theta_{c r}\end{array}\right.$

In the limiting case of a vertical wall, under ideally smooth $\left(\phi_{w} \rightarrow 0\right)$ and rough $\left(\phi_{w} \rightarrow\right.$ $\left.\phi_{w, c r} \leq \phi_{i}\right)$ the $K$ equation tends to two well-known results:

$$
\lim _{\substack{\beta \rightarrow 0 \\ \phi_{w} \rightarrow 0}} K=\frac{1+\sin \phi_{i}}{1-\sin \phi_{i}} \text { and } \lim _{\substack{\beta \rightarrow 0 \\ \phi_{w} \rightarrow \phi_{w, c r} \leq \phi_{i}}} K=\frac{1+\sin ^{2} \phi_{i}}{1-\sin ^{2} \phi_{i}}
$$

\section{Individual explorations of the mixed flow theory}

Individual predictions of mixed flow pressure distributions are illustrated here under purposefully limited sets of inputs. Except where otherwise indicated, a generic material was assumed with $\gamma=9 \mathrm{kN} / \mathrm{m}^{3}, \mu_{w}=0.44$ and $\phi_{i}=33.6^{\circ}$ (upper characteristic values for wheat for a smooth wall from Annex E of EN 1991-4, 2006). For each combination of inputs, the horizontal pressure distribution $p_{h e}(z)$ under mixed flow discharge was normalised by the Janssen 'great depth' value of the discharge horizontal pressure $K_{e} p_{0}$, where $p_{0}=\gamma z_{0}$ (Eq. 4). Separately, $p_{\text {he }}(z)$ was also normalised by the local Janssen value of the horizontal pressure $K_{e} p_{v c e, z}(z)$ (Eq. 5), with the maximum value of this ratio identifying the maximum horizontal overpressure $C_{h}$ (also given by Eq. $7 \mathrm{~b}$ ) at the effective transition under mixed flow. Lastly, the integral of the frictional traction under mixed flow $p_{w e}(z)=\mu_{w} p_{h e}(z)$ from the top surface to any given depth was normalised by the integral of the frictional tractions under concentric Janssen conditions $p_{w c e}=$ $\mu_{w} K_{e} p_{v c e, z}(z)$ (Eq. 5). The maximum value of this ratio is identified here as $C_{w}$ (Eq. 26, where the assumed constant $\mu_{w}$ has been cancelled out of the equation), and may be interpreted as an excess in membrane compression in the silo wall just below the effective transition as a consequence of mixed flow discharge.

$$
C_{w}=\max \left(\frac{\int_{0}^{z_{T}} K_{e, c w} p_{v c e, z}(z) d z+\int_{0}^{x_{T}} K_{e, s w} p_{v s e}(x) d x}{\int_{0}^{h_{c}} K_{e, c w} p_{v c e, z}(z) d z}\right)
$$


For each generated distribution, a check was additionally performed to confirm that vertical equilibrium (Eq. 27) at the base of the silo was satisfied to within a very small numerical tolerance $\varepsilon$ :

$$
\left|\pi r^{2} p_{v c e, x}(x=0)+\pi r^{2} p_{v s e}(x=0)+2 \pi r \mu_{w} \int_{0}^{z_{T}} p_{h c e, z}(z) d z+2 \pi r \mu_{w} \int_{0}^{x_{T}} p_{h s e}(x) d x-\gamma \pi r^{2} h_{c}\right|<\varepsilon
$$

The discharge lateral pressure ratios $K_{e, c w}$ and $K_{e, s w}$ were assumed to be the same, and defined by the expression corresponding to an 'active' stress state, namely Eq. 21a. The internal flow channel was assumed to be under 'passive' conditions, with relations given according to the equations in Section 2.3.3. The critical angle $\theta_{c r}$ was assumed to be such that the point $\mathrm{W}$ cannot rotate anti-clockwise further than the point opposite $\mathrm{A}^{\prime}$ on the Mohr's circle, such that:

$\theta_{c r}=\frac{\pi}{2}-\phi_{i}+2 \beta$

Equation 22 implies that the friction coefficient at the interface of the flowing and stationary solids corresponding to 'fully rough' conditions in a 'passive' state of failure cannot be taken as $\mu_{i}=\tan \phi_{i}$ as is classically assumed, but must instead adopt the reduced value

$\mu_{i}=\mu_{w, c r}=\frac{\sin \phi_{i} \cos \phi_{i}}{1+\sin ^{2} \phi_{i}}=\left(\frac{\cos ^{2} \phi_{i}}{2-\cos ^{2} \phi_{i}}\right) \tan \phi_{i}<\tan \phi_{i}$

though it is stressed that $\mu_{i}=\tan \phi_{i}$ is still achievable under 'active' conditions (Fig. 8a). For $\phi_{i}=33.6^{\circ}, \mu_{i}=\mu_{w, c r} \approx 0.53 \tan \phi_{i}$, and the angle $\omega$ (Eq. 23) plays no further role in the calculations for the flow channel. The discharge wall pressure ratio $F_{e}$ for the internal flow channel is taken to be that for an ideally-rough inclined interface under 'passive' stress conditions, namely Eq. 25b. With this choice of $\theta_{c r}$, Eq. $25 \mathrm{~b}$ yields a less onerous $F_{e}$ value, namely:

$$
F_{e}=\frac{1+\sin ^{2} \phi_{i}}{1-\sin \phi_{i} \sin \left(\phi_{i}-2 \beta\right)}
$$


In the first two explorations, the material properties were maintained unchanged while the geometry of the system was varied. In the first case, the aspect ratio was held constant at a slender value of $h_{c} / d_{c}=6$ and the depth of the effective transition was varied from $z_{T} / h_{c}=0.1$ to 0.7 in increments of 0.2 . The pressure distributions (Fig. 9) translate to loadings which would cause a significant local rise in both the circumferential tension (up to threefold under these conditions) and meridional compression (up to twofold) in the shell wall in the vicinity of the effective transition. The highest relative overpressures are predicted to occur in the top regions of the silo and decrease as the depth of the effective transition increases. Although the absolute pressures are smaller in these regions the optimised shell wall is also thinner and potentially more susceptible to large sudden changes, particularly in meridional compression. In the second case, the depth of the effective transition was held constant at a slender value of $z_{T} / h_{c}=0.3$ and the aspect ratio was varied from $h_{c} / d_{c}=2.5$ to 10 (very slender). The predicted distributions (Fig. 10) suggest that the horizontal overpressure $C_{h}$ may increase significantly with aspect ratio at constant $z_{T} / h_{c}$, from $C_{h} \approx$ 1.98 for $h_{c} / d_{c}=2.5$ to $C_{h} \approx 3.39$ at $h_{c} / d_{c}=10$, making the consequences for mixed flow potentially significantly more severe for slender silos. Changes in $C_{w}$ are more modest but still substantial, with the value remaining between 1.33 and 1.4 in this range.
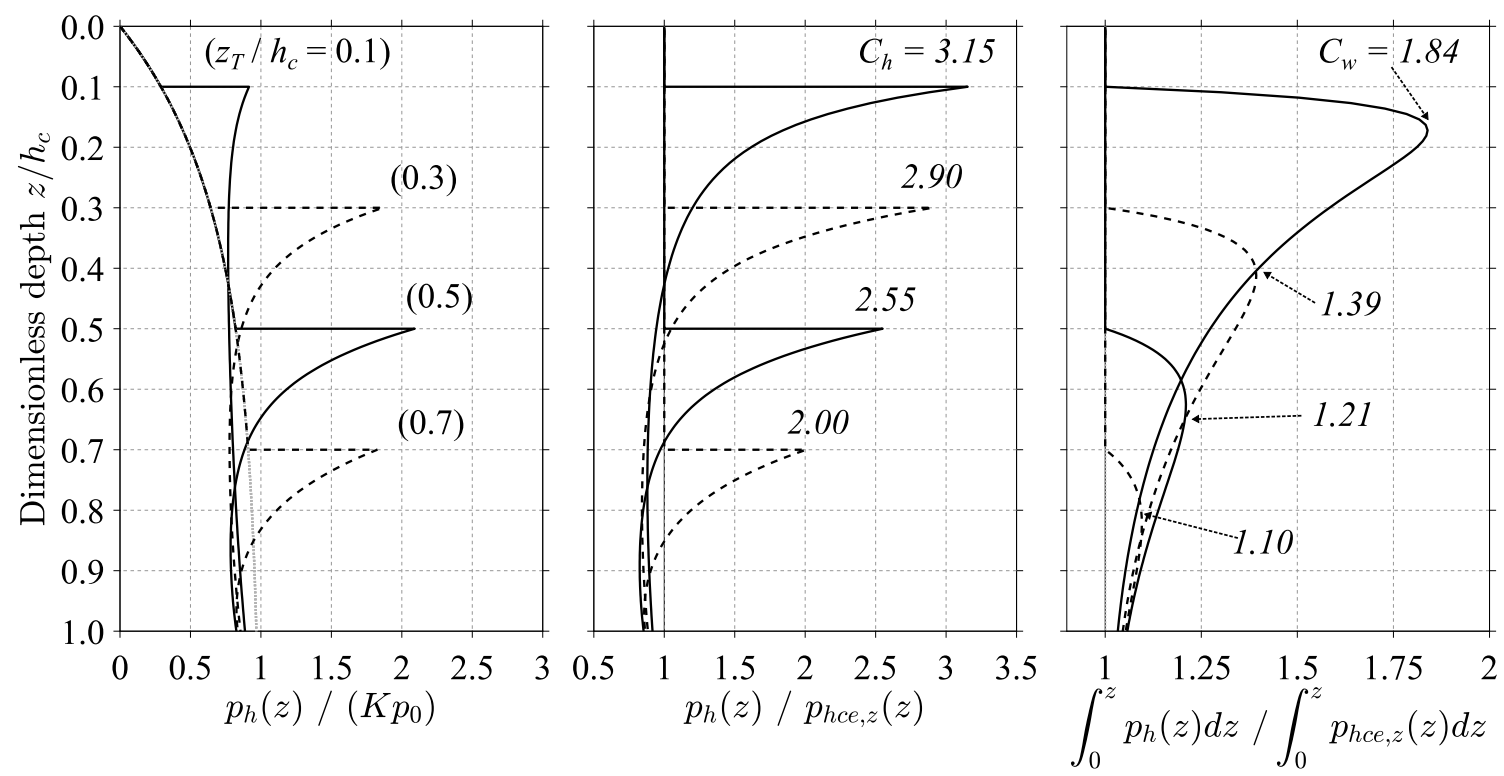

Fig. 9 - Predicted dimensionless pressure distributions at constant $h_{c} / d_{c}=6, \mu_{w}=0.44, \phi_{i}$

$$
=33.6^{\circ} \text { and varying } z_{T} / h_{c} \text {. }
$$



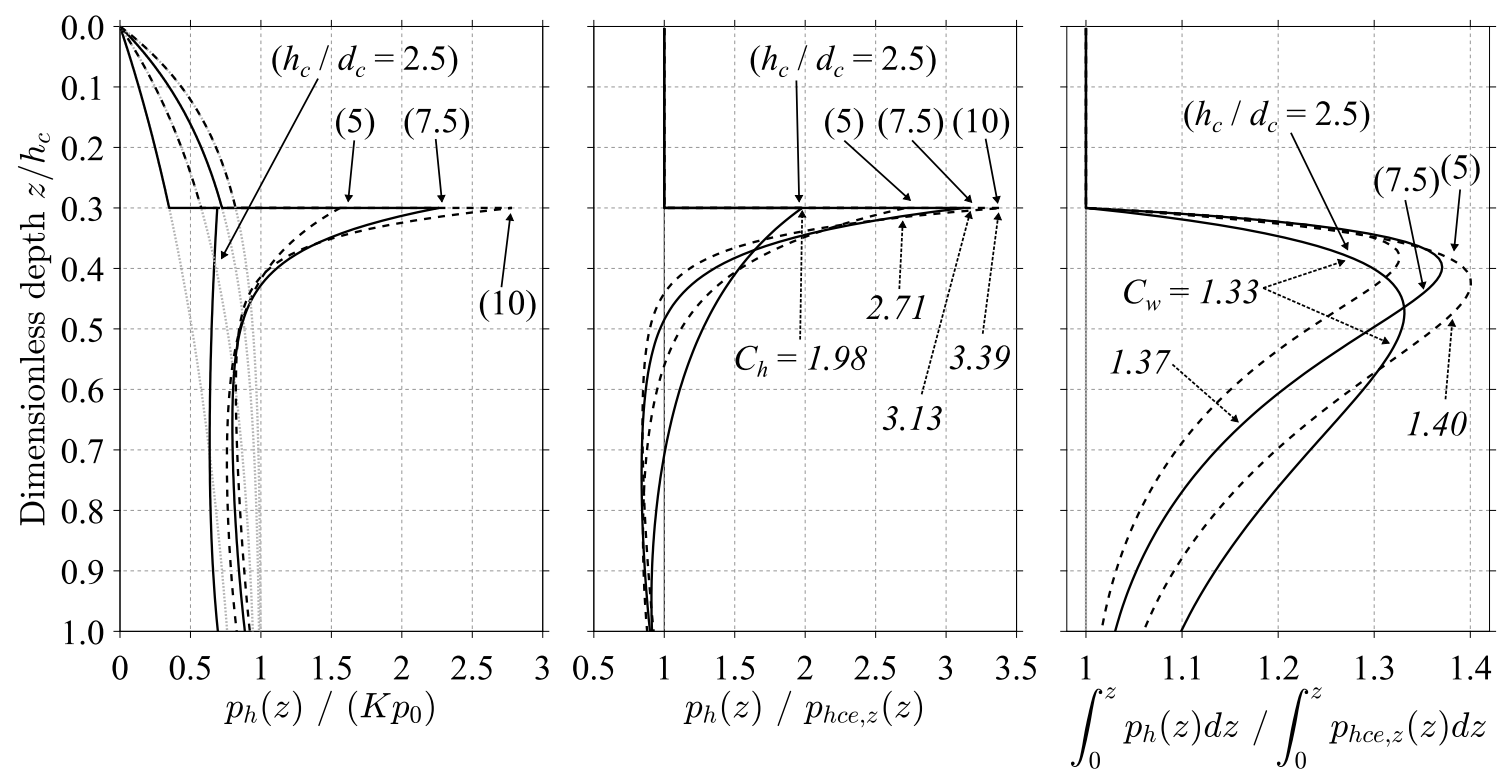

Fig. 10 - Predicted dimensionless pressure distributions at constant $z_{T} / h_{c}=0.3, \mu_{w}=0.44$,

$$
\phi_{i}=33.6^{\circ} \text { and varying } h_{c} / d_{c} \text {. }
$$

In the next two explorations, the geometry of the system was maintained unchanged while the material properties were varied. Firstly, the silo wall friction coefficient $\mu_{w}$ was varied so as to achieve a wall friction angle $\phi_{w}$ ranging from a very smooth wall $\left(\phi_{w}\right.$ $\left./ \phi_{i}=0.1\right)$ to an 'ideally rough' wall $\left(\phi_{w} / \phi_{i}=1\right)$, assuming that the solid against the silo wall remained in a state of 'active' failure. The predicted distributions (Fig. 11) may be interpreted to suggest that smoother walls lead to significantly higher rises in both circumferential tension (up to fivefold under these conditions) and meridional compression (up to twofold) at the effective transition. Secondly, the silo wall friction coefficient was kept constant at $\mu_{w}=0.44\left(\phi_{w} \approx 23.5^{\circ}\right)$ and the internal friction angle was varied from $\phi_{i}=25^{\circ}$ to $45^{\circ}$ in increments of $5^{\circ}$, such that $\phi_{w}<\phi_{i}$. The pressure distributions (Fig. 12) in this case suggest that stronger solids may exhibit higher overpressures at the transition, with $C_{h}$ and $C_{w}$ ranging from $\sim 1.45$ and $\sim 1.07$ for $\phi_{i}=$ $25^{\circ}$ to $\sim 4.22$ and $\sim 1.45$ for $\phi_{i}=45^{\circ}$ respectively. The larger peak pressure for stronger materials has been observed in several tests (Nielsen and Andersen, 1981; Nielsen, 1998). In all distributions shown, the entire portion of the shell below the effective transition suffers a rise in meridional compression, with the peak consistently occurring just below it. 

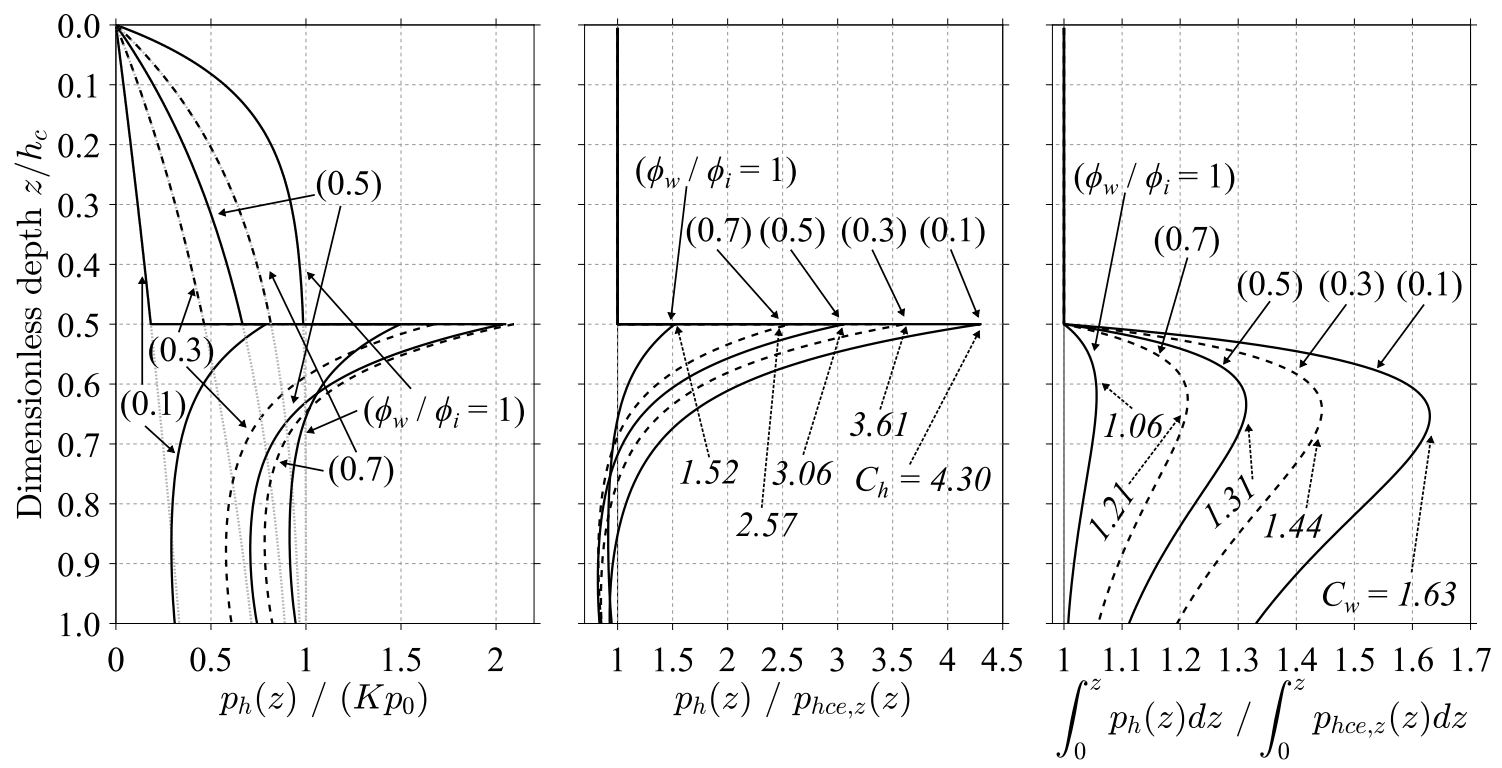

Fig. 11 - Predicted dimensionless pressure distributions at constant $h_{c} / d_{c}=6, z_{T} / h_{c}=0.5$, $\phi_{i}=33.6^{\circ}$ and varying $\phi_{w} / \phi_{i}$.
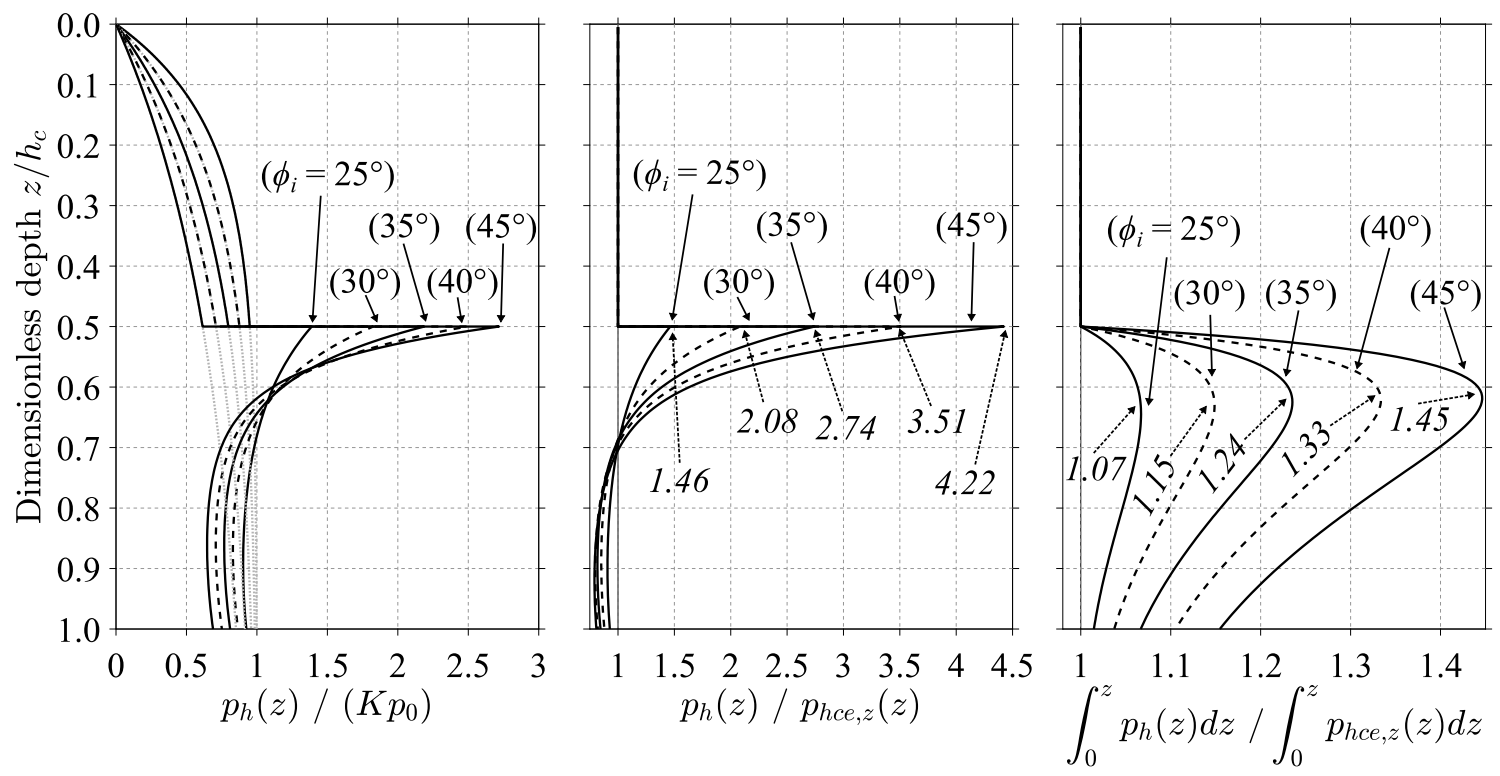

Fig. 12 - Predicted dimensionless pressure distributions at constant $h_{c} / d_{c}=5, z_{T} / h_{c}=0.5$, $\mu_{w}=0.44$ and varying $\phi_{i}$. 


\section{Global parametric investigation}

A comprehensive parametric investigation is offered here to illustrate the predictions of the mixed flow pressure theory across an exhaustive range of all realistically possible inputs. Each individual predicted pressure distribution was characterised by a select and compact group of dimensionless variables:

- $C_{h}$, the horizontal overpressure at the effective transition (Eq. $7 \mathrm{~b}$ );

- $C_{w}$, the maximum value of the ratio of the integral of the frictional wall tractions at any depth under mixed flow relative to what it would have been under mass flow (Eq. 26). This is the excess in accumulated wall shears and induced membrane compression in the silo wall;

- $F_{t}$, the ratio of the net outward force under mixed flow (obtained by numerically integrating the horizontal pressure distribution from the effective transition to the 'crossover' depth, see the definition for $S_{t}$ below) to the force that would have been present under mass flow integrated over the same region;

- $G_{t}$, the ratio of the gradient of the horizontal pressures at the effective transition relative to what it would have been under mass flow (Eq. 15);

- $S_{t}$, the depth of the intersection of the horizontal pressure distribution under mixed flow with the Janssen distribution under mass flow below the effective transition (the 'crossover' depth) normalised by the silo diameter.

The following complete parameter ranges were investigated:

- Aspect ratios $h_{c} / d$ from 1 to 5 in increments of 0.2 , assuming a unit radius $r$;

- Effective transition positions $z_{T} / h_{c}$ from 0.1 (almost pipe flow) to 0.8 (almost mass flow) in increments of 0.05 ;

- Silo wall friction coefficients $\mu_{w}$ from 0.2 (smooth) to 0.6 (rough) in increments of 0.05 ;

- Internal friction angles $\phi_{i}$ from $20^{\circ}$ (weak) to $40^{\circ}$ (strong) in increments of $2^{\circ}$.

All permutations of the above were considered as long as they satisfied the conditions that $\mu_{w} \leq \tan \phi_{i}$ at the silo wall as well as Eqs 23 and 24. Both assumptions for $\theta_{c r}$ (Eq. 24) were investigated, labelled $\theta_{c r, 1}$ and $\theta_{c r, 2}$ respectively, leading to two data sets of 
approximately 10,500 solutions each. As the mixed flow theory is linear in the unit weight $\gamma$, a single representative constant value of $10 \mathrm{kN} / \mathrm{m}^{3}$ was chosen in all cases. The process was controlled using the SIMULIA Isight (2017) automation software.

Given the large number of results, the descriptive dimensionless variables are presented in the form of frequency histograms in Fig. 13 which offer a global overview of the distribution of likely values. Linear correlation coefficients of these variables against the input variables are offered in Table 1, reflecting many of the same tendencies globally as those identified in Section 3 for individual distributions. The $C_{h}$ variable exhibits a significant right skew and appears to follow a log-normal distribution, suggesting that under most combinations of uniformly-sampled geometry and material inputs the most probable overpressure at the effective transition is approximately 1.8 times the local (filling) Janssen pressure. Globally, $C_{h}$ exhibits a medium-strength positive correlation with aspect ratio and effective transition depth (contrary to that shown in Fig. 9), and a negative correlation with the internal friction angle. Some very high overpressures are possible, but these correspond to increasingly unlikely combinations. Similarly, the strongly right-skewed $C_{w}$ variable suggests that the most likely increase in meridional membrane compression in the silo wall will be approximately $10 \%$ under these conditions, though some less likely combinations of inputs can be more severe. $C_{w}$ is globally strongly negatively correlated with the internal friction angle.

The strong left skew of the $G_{t}$ variable histograms suggests that almost all predicted pressure patterns will exhibit a sharp pressure peak at the effective transitions followed by a steep descent (left-hand side of Fig. 6). Indeed, $G_{t}$ is negatively correlated with all input variables. The symmetrically-distributed $S_{t}$ variable suggest that most of these steeply-descending pressure patterns will most probably intersect with the hypothetical Janssen distribution at a depth of approximately one diameter below the effective transition. This 'crossover' depth is likely to increase with the aspect ratio, but decrease with the internal friction angle. The similarly symmetrically-distributed $F_{t}$ variable points to a most probable net increase of the net radial force applied to the silo wall of $\sim 30 \%$ under mixed flow, and exhibits only weak correlations with the input variables. Lastly, the predictions of the concentric mixed flow theory do not appear to be very sensitive to the choice of critical angle $\theta_{c r}$ assumed here, and changing between these values does not cause a qualitative change in the tendencies described above. 


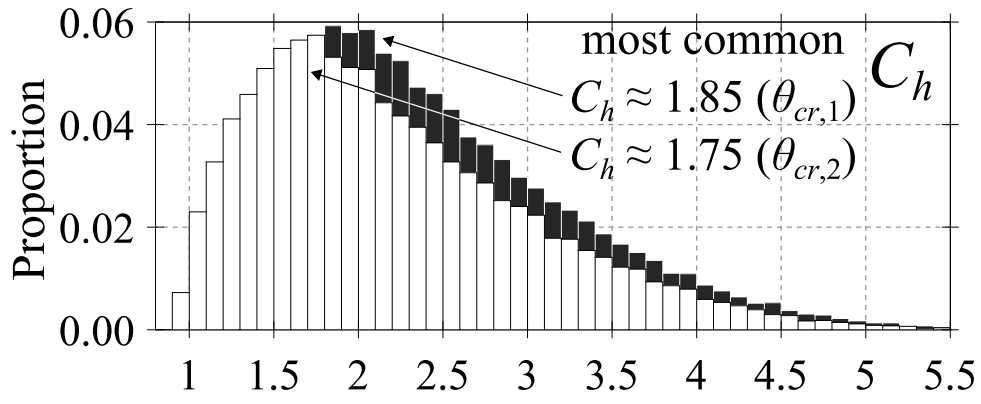

$\theta_{c r}, 1$

min: 1.16

$\max : 6.12$

mean: 2.48

std: 0.80

skew: 0.92

$\theta_{c r, 2}$

min: 0.93

$\max : 5.94$

mean: 2.22

std: 0.82
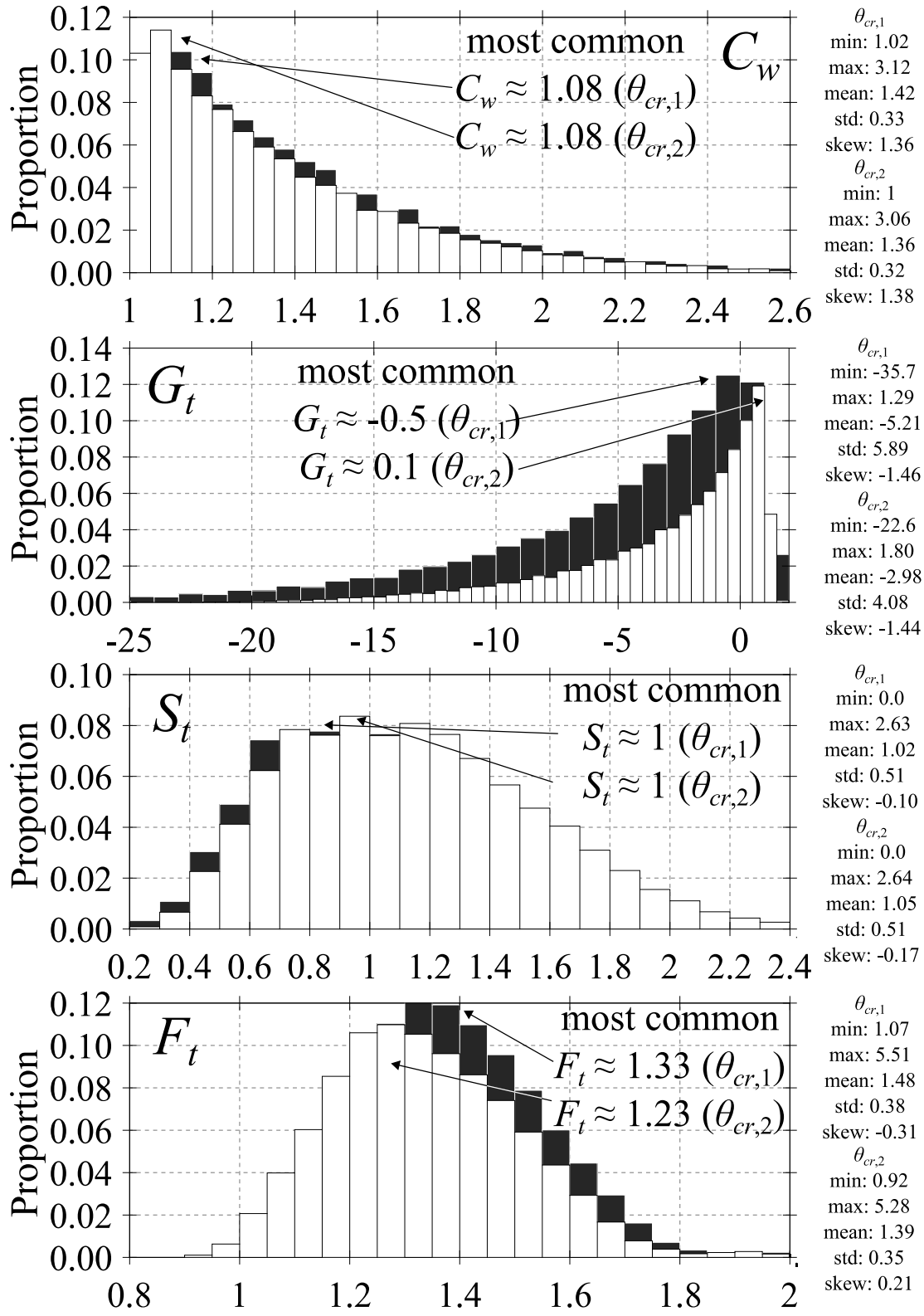

$\theta_{c r, 1}$

min: 1.07

$\max : 5.51$

mean: 1.48

std: 0.38

skew: -0.31

$\theta_{c r, 2}$

min: 0.92

max: 5.28

mean: 1.39

std: 0.35

skew: 0.21

Fig. 13 - Histograms and summary statistics of the $C_{h}, C_{w}, G_{t}, S_{t}$ and $F_{t}$ dimensionless variables for two expressions for $\theta_{c r}$. 
Table 1 - Linear correlation coefficients for $C_{h}, C_{w}, G_{t}, S_{t}$ and $F_{t}$ dimensionless variables against the dimensionless inputs $h_{c} / d_{c}, z_{T} / h_{c}, \mu_{w}$ and $\phi_{i}$.

\begin{tabular}{|c|c|c|c|c|}
\hline & $h_{c} / d_{c}$ & $z_{T} / h_{c}$ & $\mu_{w}$ & $\phi_{i}$ \\
\hline$C_{h}$ & 0.43 & 0.50 & 0.16 & -0.64 \\
\hline$C_{w}$ & 0.19 & 0.34 & 0.04 & -0.82 \\
\hline$G_{t}$ & -0.65 & -0.41 & -0.39 & -0.31 \\
\hline$S_{t}$ & 0.40 & -0.02 & 0.02 & -0.64 \\
\hline$F_{t}$ & 0.24 & 0.31 & 0.12 & -0.32 \\
\hline
\end{tabular}

\section{Discussion}

The theory, as described above, offers a rigorous treatment of mixed flow pressures allowing for studies of influence by all parameters necessary for design as in EN 1991-4 (2006). Reference has been made to experiments, which show that important phenomena are reflected such as a significant redistribution of pressure during discharge, most significantly that the redistribution potential (difference between maximal filling pressure and maximal discharge pressure) increases with an increase in the internal angle of friction of the stored material. This means that, opposite to the prediction of the widely used Janssen theory (1895), silos exposed to mixed flow experience more demanding loads the higher the internal angle of friction of the stored material.

However, for a practical use of the theory, a number of considerations should be kept in mind. Firstly, materials that are known to develop cohesion, such as cement, flour, soya meal and powders, have not been considered. However, powder containers are generally designed to achieve mass flow and thus mixed flow is unlikely to occur. Secondly, granular materials consisting of particles with one or more dimensions significantly larger than any other, such as wood chips, are unsuitable for treatment as a Coulombic material (Nielsen, 1998). Additionally, highly anisotropic granular materials cannot be guaranteed to be free-flowing under gravity, and their handling requires specialist equipment and design procedures (EN 1991-4, 2006). Lastly, the conical form of the internal flow channel is an assumption taken for mathematical convenience in 
formulating the exploratory differential equations, although it is a reasonable representation of channels based on experimental observations (Nielsen and Askegaard, 1977). An extension of the present theory for flow channels of arbitrary cross-section, a powerful tool to explore the sensitivity of the pressure regime to the assumed flow pattern, will be presented at a later time. It should be added that two key hypotheses of this theory, the dimensionless overpressure and the treatment of the passive stress state under ideally rough conditions, are independent of the profile of the flow channel.

Direct experimental verification of the phenomena described in this paper is to this day scarce and what historical data exists indicates a more complex behaviour than can be captured through equilibrium treatments alone. Embedded pressure cells have been used to obtain information about horizontal and vertical stresses in the plug flow zone in a silo with mass flow (Munch-Andersen and Nielsen, 1989; 1990). These experiments indicate that the transition takes place in a zone above and below the transition plane assumed by the theory. This means that the magnitude of the theoretical peak pressure as well as the precise distribution of the flow pressure may differ from the predicted value. This justifies the use of an empirical factor on the peak pressure to be introduced in Standards to become in line with safety margins which have served well.

\section{Conclusions}

This paper has presented an equilibrium-based algebraic theory to predict the silo wall pressures under the idealised condition of a fully-developed mixed flow pattern with the silo in a filled state. This 'mixed flow theory' offers a rigorous basis for the algebraic evaluation of loads in mixed flow silos for the first time, and is recommended to be used as a basis for forming load models for silos with mixed flow.

A careful interpretation of the predictions of the theory was performed in the context of the parameters used in EN 1991-4 (2006), and limitations for practical use have been discussed. Further experimental verification is needed, particularly regarding:

- Flow pattern and resulting wall pressure observations in full-scale silos;

- Verification of the stress state adjacent to the wall in the 'plug flow' region above the effective transition; 
- Detailed descriptions of pressure distributions near the effective transition;

- Careful verification of the stress regime in the rupture layer in rough-walled silos under passive conditions.

The authors suggest that the theory developed in this paper may be applied directly by industry as long as the results are interpreted with insight and caution.

\section{Appendix A - Location of peak axial compression in the silo wall}

The two critical metrics of the mixed flow theory for structural design are the overpressure at the effective transition $C_{h}$ and the maximal increase in frictional tractions described by $C_{w}$. Although a simple closed-form solution exists for $C_{h}$ (Eq. 7), establishing $C_{w}$ requires an onerous numerical solution to the differential equation governing the pressure in the stationary solid (Eqs $10 \& 11)$ as well as the subsequent integral (Eq. 26). This Appendix offers two closed-form procedures to approximate $C_{w}$.

\section{A.1 Partially closed-form procedure to approximate $C_{w}$}

Let the solution for the mean vertical pressure within the stationary solid is approximately expressed in terms of the $z$ coordinate as:

$p_{v s e, z}(z) \approx a_{1}+a_{2} z+\frac{a_{3}}{z^{2}}$ with coefficients $a_{1}, a_{2}$ and $a_{3}$ such that: $\left\{\begin{array}{c}p_{v s e, z}\left(z_{T}\right)=p_{v s e T} \\ \left.\frac{d p_{v s e, z}}{d z}\right|_{z=z T}=\left.\frac{d p_{v s e}}{d z}\right|_{z=z T} \\ R_{h c}=0\end{array}\right.$

where $\underline{p}_{v s e T}$ and $\left.\left(d p_{v s e} / d z\right)\right|_{z T}$ are obtained from Eqs $7 \mathrm{a}$ and $14 \mathrm{a}$ respectively, and $R_{h c}$ is the residual of the differential equation (Eq. 10) after substitution with the above functional form and set to zero at the bottom of the silo $\left(z=h_{c}\right)$. A closed-form procedure for obtaining an approximate value of $C_{w}$ and its location $z_{w}$ is as follows:

$$
C_{w} \approx \max _{\text {on } z_{T} \leq z \leq h_{c}}\left(\frac{K_{e, c w} \gamma z_{o}^{2}\left(e^{-z_{T} / z_{o}}-1+\frac{z_{T}}{z_{o}}\right)+K_{e, s w}\left[a_{1}\left(z-z_{T}\right)+\frac{a_{2}}{2}\left(z^{2}-z_{T}^{2}\right)-a_{3}\left(\frac{1}{z}-\frac{1}{z_{T}}\right)\right]}{K_{e, c w} \gamma z_{o}^{2}\left(e^{-z / z_{o}}-1+\frac{z}{z_{o}}\right)}\right)
$$


where

$$
\begin{aligned}
& a_{1}=\frac{1}{a_{4}}\left(\left(2 h_{c}^{3}+z_{T}^{3}\right) p_{v s e T}+\left.\left(h_{c}^{3}-z_{T}^{3}\right) z_{T} \frac{d p_{v s e}}{d z}\right|_{z T}-3 h_{c}^{2} z_{T} p_{v s e B}\right) \\
& a_{2}=\frac{1}{a_{4}}\left(2 h_{c}^{2}\left(p_{v s e B}-p_{v s e T}\right)-\left.\left(h_{c}^{2}-z_{T}^{2}\right) z_{T} \frac{d p_{v s e}}{d z}\right|_{z T}\right) \\
& a_{3}=\frac{1}{a_{4}} h_{c}^{2} z_{T}^{3}\left(p_{v s e B}-p_{v s e T}-\left.\left(h_{c}-z_{T}\right) \frac{d p_{v s e}}{d z}\right|_{z T}\right) \\
& a_{4}=\left(2 h_{c}+z_{T}\right)\left(h_{c}-z_{T}\right)^{2} \\
& p_{v s e B}=\frac{\frac{1}{2}\left(h_{c}\left(2 h_{c}+z_{T}\right) \gamma+\left.z_{T}\left(h_{c}+2 z_{T}\right) \frac{d p_{v s e}}{d z}\right|_{z T}\right)\left(h_{c}-z_{T}\right)+\left(h_{c}^{2}+h_{c} z_{T}+z_{T}^{2}\right) p_{v s e T}}{(2 m+1) h_{c}^{2}+(m+1) h_{c} z_{T}+z_{T}^{2}}
\end{aligned}
$$

Identifying $C_{w}$ and the depth $z_{w}$ below the reference surface at which it occurs requires an inspection of a plot of Eq. A.1 within the range $z_{T} \leq z \leq h_{c}$. The accuracy of $C_{w}$ and $z_{w}$ established in this manner is illustrated in Fig. A.1.
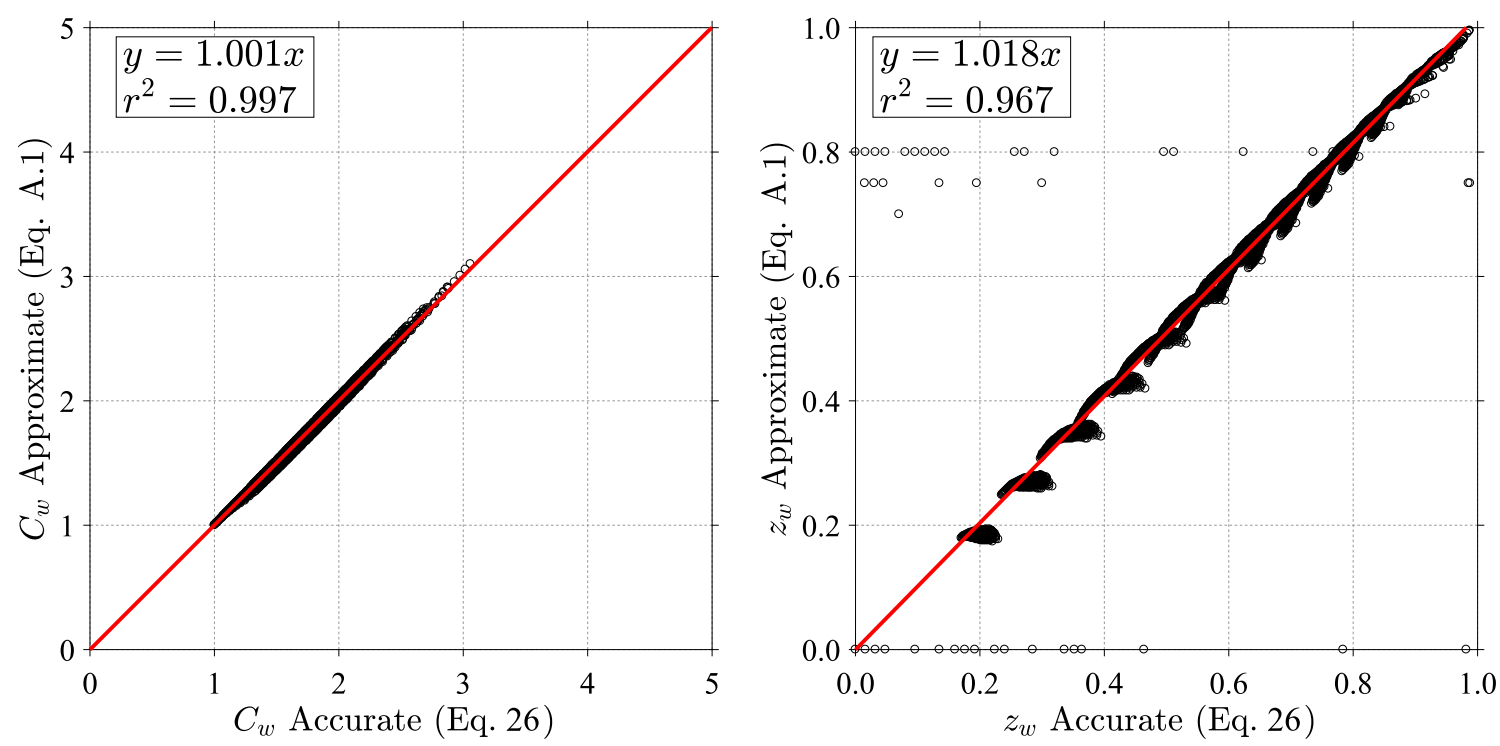

Fig. A.1 - Scatterplot of accurate and approximate $C_{w}$ and its location $z_{w} / h_{c}$, established by identifying the maximum of a plotted distribution (Eq. A.1). 
A.2 Fully closed-form procedure to approximate $C_{w}$

A fully closed-form approximation for the location of $C_{w}$ is as follows (assuming $K_{e, c w}=$ $\left.K_{e, s w}\right)$ :

$z_{w} \approx \frac{\sum_{j=0}^{2} c_{1 j} \cdot e^{-j \cdot z_{T} / z_{o}}+\sqrt{\sum_{j=0}^{4} c_{2 j} \cdot e^{-j \cdot z_{T} / z_{o}}}}{\sum_{j=0}^{2} c_{3 j} \cdot e^{-j \cdot z_{T} / z_{o}}}$ but no less than $z_{T}$ and no greater than $h_{c}$

where

$$
\begin{aligned}
& c_{10}=-\left[\left(4 z_{T}^{4}-3 z_{o} z_{T}^{3}\right) a_{2}-2 a_{3} z_{T}\right] z_{o} \\
& c_{11}=\left[\left(a_{2}-\gamma\right) z_{T}^{4}+\left(\left(a_{2}+4 \gamma\right) z_{o}+a_{1}\right) z_{T}^{3}-3 z_{o}^{2}\left(a_{2}+\gamma\right) z_{T}^{2}+a_{3}\left(z_{T}-2 z_{o}\right)\right] z_{T} \\
& c_{12}=\left(3 z_{o}-z_{T}\right) \gamma z_{o} z_{T}^{3} \\
& c_{20}=\left[\begin{array}{c}
5 a_{2}^{2} z_{T}^{6}-3 a_{2}\left(\left(a_{2}+2 \gamma\right) z_{o}-2 a_{1}\right) z_{T}^{5}+2\left(5 z_{T}-6 z_{o}\right) a_{2} a_{3} z_{T}^{2} \ldots \\
-4\left(a_{1}-\gamma z_{o}\right)\left(a_{2} z_{T}^{3}+a_{3}\right) z_{o} z_{T}-4 a_{3}^{2}
\end{array}\right]\left(z_{o}-z_{T}\right) z_{o}^{2} z_{T} \\
& c_{21}=\left[\begin{array}{c}
-2 a_{2}\left(\gamma-a_{2}\right) z_{T}^{9}+2\left(\left(2 a_{2}+\gamma\right) \gamma z_{o}+a_{1}\left(2 a_{2}-\gamma\right)\right) z_{T}^{8} \ldots \\
-2\left(\left(5 a_{2}^{2}+6 a_{2} \gamma+4 \gamma^{2}\right) z_{o}^{2}+a_{1}\left(a_{2}-3 \gamma\right) z_{o}-a_{1}^{2}\right) z_{T}^{7} \ldots \\
+2\left(\left(3 a_{2}^{2}+12 a_{2} \gamma+5 \gamma^{2}\right) z_{o}^{3}-2 a_{1}\left(3 a_{2}+2 \gamma\right) z_{o}^{2}-a_{1}^{2} z_{o}+a_{3}\left(2 a_{2}-\gamma\right)\right) z_{T}^{6} \ldots \\
-2\left(2\left(3 a_{2}+\gamma\right) \gamma z_{o}^{4}-2 a_{1}\left(2 a_{2}+\gamma\right) z_{o}^{3}+\left(3 a_{2}-5 \gamma\right) a_{3} z_{o}-2 a_{1} a_{3}\right) z_{T}^{5} \ldots \\
-4\left(\left(5 a_{2}+3 \gamma\right) z_{o}+2 a_{1}\right) a_{3} z_{o} z_{T}^{4}+2\left(6\left(2 a_{2}+\gamma\right) z_{o}^{3}+a_{3}\right) a_{3} z_{T}^{3} \ldots \\
+2\left(2\left(2 a_{1}-3 \gamma z_{o}\right) z_{o}^{2}-3 a_{3}\right) a_{3} z_{o} z_{T}^{2}+8 a_{3}^{2} z_{o}^{2} z_{T}
\end{array}\right] z_{o} \\
& c_{22}=\left[\begin{array}{c}
\left(2 a_{2}^{2}-2 a_{2} \gamma-\gamma^{2}\right) z_{T}^{8}+2\left(\left(a_{2}^{2}+3 a_{2} \gamma+5 \gamma^{2}\right) z_{o}-a_{1} \gamma+2 a_{1} a_{2}\right) z_{T}^{7} \ldots \\
-\left(3\left(a_{2}^{2}+6 \gamma a_{2}+7 \gamma^{2}\right) z_{o}^{2}-2 a_{1}\left(a_{2}+4 \gamma\right) z_{o}-2 a_{1}^{2}\right) z_{T}^{6} \ldots \\
+2\left(6\left(a_{2}+\gamma\right) \gamma z_{o}^{3}-2 a_{1}\left(a_{2}+2 \gamma\right) z_{o}^{2}+a_{3}\left(2 a_{2}-\gamma\right)\right) z_{T}^{5} \ldots \\
-2\left(\left(a_{2}-6 \gamma\right) a_{3} z_{o}-2 a_{1} a_{3}\right) z_{T}^{4}-4\left(3\left(a_{2}+\gamma\right) z_{o}+a_{1}\right) a_{3} z_{o} z_{T}^{3} \ldots \\
+2\left(a_{3}-2 a_{1} z_{o}^{2}+6 \gamma z_{o}^{3}\right) a_{3} z_{T}^{2}-4 a_{3}^{2} z_{o} z_{T}
\end{array}\right] z_{o}^{2} \\
& c_{23}=-2\left[\gamma^{2} z_{T}^{7}-2\left(\left(a_{2}+3 \gamma\right)\left(z_{T}-z_{o}\right)+a_{1}\right) \gamma z_{o} z_{T}^{5}-2\left(z_{T}^{2}-z_{o} z_{T}\right) a_{3} \gamma z_{o} z_{T}\right] z_{o}^{3}
\end{aligned}
$$




$$
\begin{aligned}
& c_{24}=\left(4 z_{o}-z_{T}\right) \gamma^{2} z_{o}^{4} z_{T}^{5} \\
& c_{30}=\left[-7 a_{2} z_{T}^{3}+2\left(\left(3 a_{2}+\gamma\right) z_{o}-a_{1}\right) z_{T}^{2}+2\left(a_{1}-\gamma z_{o}\right) z_{o} z_{T}+2 a_{3}\right] z_{o} \\
& c_{31}=\left(a_{2}-\gamma\right) z_{T}^{4}+\left(\left(a_{2}+5 \gamma\right) z_{o}+a_{1}\right) z_{T}^{3}-6\left(a_{2}+\gamma\right) z_{o}^{2} z_{T}^{2}+\left(a_{3}-2 a_{1} z_{o}^{2}+4 \gamma z_{o}^{3}\right) z_{T}-2 a_{3} z_{o} \\
& c_{32}=-\left(2 z_{o}^{2}-4 z_{o} z_{T}+z_{T}^{2}\right) \gamma z_{o} z_{T}
\end{aligned}
$$

and $a_{1}, a_{2}$ and $a_{3}$ are as given by Eqs A.2 to A.4. Introducing $z_{w}$ as $z$ in Eq. A.1 will allow the direct approximation of $C_{w}$. These coefficients were established with the aid of a Taylor series expansion of the derivative of the contents of the brackets in Eq. A.1 about $z=z_{T}$ and the isolation of the appropriate root which identifies $z_{w}$. The accuracy of $C_{w}$ and $z_{w}$ established in this manner is illustrated in Fig. A.2.
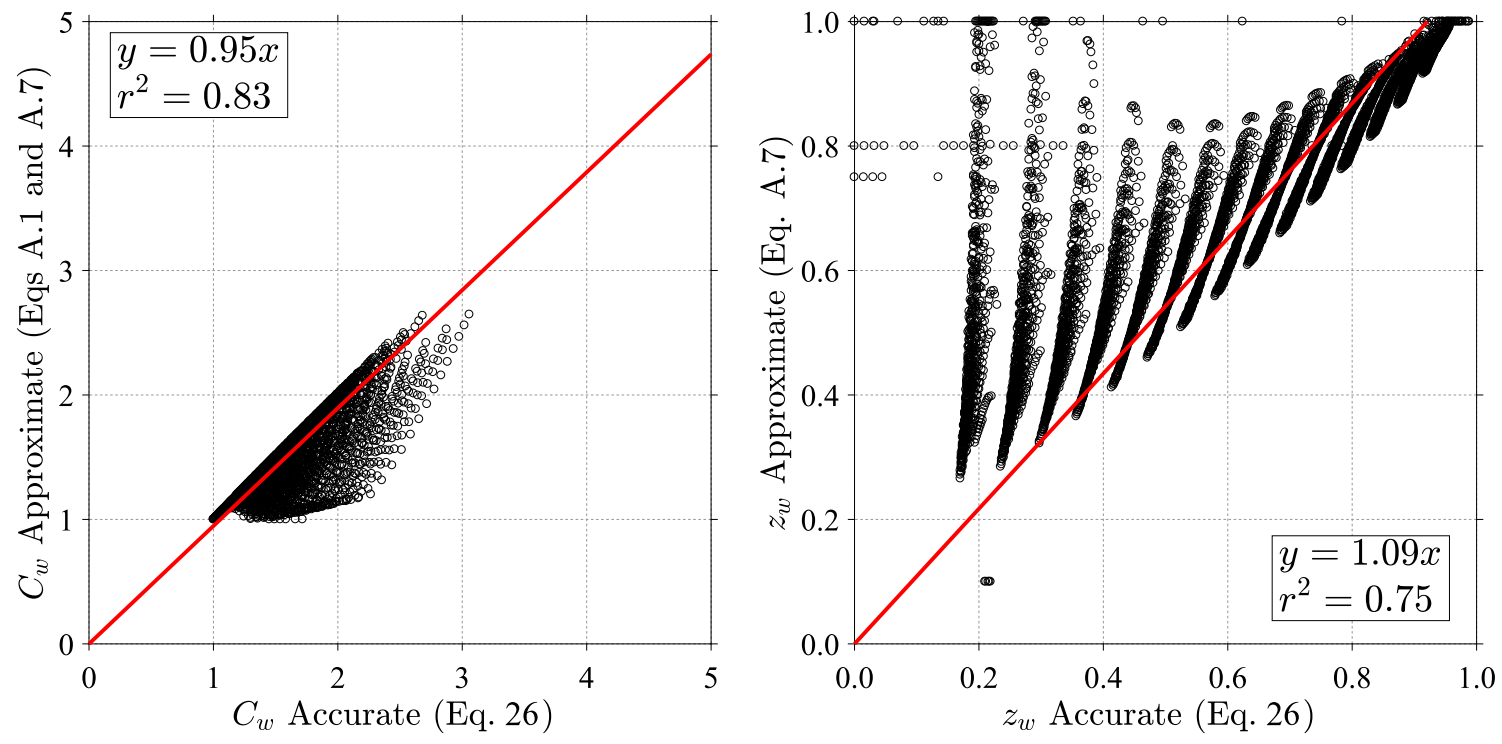

Fig. A. 2 - Scatterplot of accurate and approximate $C_{w}$ and its location $z_{w} / h_{c}$, established by a closed-form expression (Eqs A.1 \& A.7).

\section{References}

Dąbrowski A. (1957). "Parcie Materiałów Sypkich w Leju (Pressures from Bulk Solids in Hoppers)." Archiwum Inżynierii Ladowej, Warsaw, Poland, Z.3, 325-328.

Drescher A. (1991) “Analytical methods in bin-load analysis” Elsevier, New York. 
EN 1991-4 (2006) "Eurocode 1: Actions on Structures, Part 4: Silos and Tanks." European Committee for Normalisation, Brussels.

Hampe E.H. (1987) "Silos Band 1: Grundlagen” VEB Verlag für Bauwesen, Berlin, GDR.

Hartlén et al (1984) "The wall pressure in large grain silos." Swedish Council for Building Research, D2:1984

Isight (2017) "Isight 2017 Process Control and Automation Software and Documentation" Dassault Systèmes, Simulia Corporation, Providence, RI, USA.

Janssen H.A. (1895) "Versuche über Getreidedruck in Silozellen" Zeitschrift des Vereines Deutcher Ingenieure, 39(35), 1045-1049.

Jenike A.W. (1961) "Gravity Flow of Bulk Solids" Bull. 108, Utah Engineering Experiment Station, University of Utah, USA.

Jenike A.W. (1964) "Storage and Flow of Bulk Solids" Bull. 123, Utah Engineering Experiment Station, University of Utah, USA.

Khelil A. (1989) "Etude du champs de vitesses et de constraints dans les silos métalliques" Thesis, INPL de Nancy.

Khelil A. \& Roth J.C. (1990) "Spécification des charges écoulements dans les silos métalliques" Revue Française de Géotechnique, 52, 11-25.

Munch-Andersen J. \& Nielsen J. (1989) "The internal pressures in the outlet zone and the transition zone of a silo." 3rd Int. Conf. On Bulk Materials, Handling and Transportation, 27-29 June 1989. The Institution of Engineers, Newcastle, NSW, Australia.

Munch-Andersen J. \& Nielsen J. (1990) "Pressures in slender grain silos - Measurements in three silos of different sizes." CHISA `90, 26-31 august, 1990, Praha, Check Republic.

Nedderman R.M. (1992) "Statics and kinematics of granular materials" Cambridge University Press, UK.

Nielsen J. (1998) "Pressures from flowing granular solids in silos." Phil. Trans. Royal Society, London. A (1998) 356, 2667-2684.

Nielsen J. and Askegaard V. (1977) "Scale errors in model tests on granular media with special reference to silo models" Powder Technology, 16(1), 123-130.

Nielsen J. and Andersen E.Y. (1981) "Loads in Grain Silos". 11th working session of the IAAS Committee of pipes and tanks, Wrocław, November.

Rotter J.M. (2001) "Guide for the economic design of circular metal silos" Spon Press.

Saleh K., Golshan S. \& Zarghami R. (2018) "A review on gravity flow of free-flowing granular solids in silos - Basics and practical aspects" Chemical Engineering Science, 192, 1011-1035.

Walker D.M. (1966). "An Approximate Theory for Pressure and Arching in Hoppers." Chemical Engineering Science, 21, 957-997.

Walters J.K. (1973). "A theoretical analysis of stresses in silos with vertical walls." Chemical Engineering Science, 28, 13-21.

Zhong Z., Ooi J.Y. \& Rotter J.M. (2001) "The sensitivity of silo flow and wall stresses to filling method" Engineering Structures, 23, 756-767. 